\title{
Alexander Roßnagel Das Recht auf (tele-)kommunikative Selbstbestimmung
}

\section{ISDN: Kommunikationstechnik - cin Rechtsproblem?}

Für den Weg in die informationsgesellschafu gits die derzeitige Telckommunikationsinfrastruktur als unzureichend. Die erstrebte Informatisierung fast aller Lebensberciche erscheint auf ihrer Grundlage unmöglich. Zwar ist die Anschlußdichte des Fernsprechnetzes mit seinen bundesweit ca. 28 Millionen Teilnehmern relativ hoch. Auch werden im Fcrnsprechnecz neben dem. Tclefondienst auch Datenübertragungsdienste und der Telefaxdienst (für das Fernkopieren) angeboren. Doch sind der Nutzung dieses Netzes zu anderen Zwecken als dem Telefonieren enge Grenzen gesetzt. In ihm ist die Daten-, Bild-oder Textkommunikacion für professionelle Zwecke zu aufwendig', zu langsam² und damir zu wenig wirtschafelich. Daher wird der uberwiegende Teil des Texr- und Datenverkehrs über sperielle Darennerze abgewickelt. Dies sind das Datex-L-Nerz, das Datcx-P-Nerz und Standleitungen (Direktrufnctz), in ihrer Gcsamcheic "Integriertes Dutenneliz (IDN) genannt. Neben verschiedenen Datenübertragungsdiensten werden über das IDN auch Telex (Fernschrciben) und Telctex (Bürofernschreiben) angeboren. Diese Dienste sind vergleichsweise zeuer. ${ }^{3}$ Außerdem ist für jeden Diensc eine getrennte Anschlußleitung und cine eigene Rufnummer erforderlich. Übergänge von einem zum anderen Teilnecz sird nur mic großem Aufwand möglich. ${ }^{+}$Besonders nachteilig ist jedoch, daß im IDN nicht relefonicrt werden kann. Daher sind dic Haushalte über dic Datennetze niche zu erreichen.'

Diese Begrenzungen werden jedoch erst als Nachteil begriffen, seit es das Ziel von Unternehmen und Organisationen ist, umfangreiche Compucernetzwerke aufzubauen und den großen Markt der ctwa as Millionen Haushalce einzubeziehen. ${ }^{6}$ Erst

- Der Teılnehmer benougı spezsclle zusialiche Fndgerate. dic die digutalen Signale der Fernkuprerer oder Dasenverarbeicungsanlagen in analoge Signale verwandeln (modulicren) und auf der Gegenseite wicder zurückvirwandeln (demodulieren) (MODEMs).

2 Die nucsbaren Übereragungsraten sind mit max. $9,6 \mathrm{kbu} / \mathrm{s}$ erbeblich mednger als die des ISDN mit $64 \mathrm{kbir} / \mathrm{s}$. Zu den Übereragungsraten s. zuch Fn. 19.

3 So betragen dic Grundgebühren für einen Djecx-L-Anschluß mat einer Übertragungsrate von 2,4 kbu/s monatlich DM i80.- und bet einer Überiragungsrate von $48 \mathrm{kbit} / \mathrm{s}$ monatlich DM 2000,-. Fur cincn Datex-P-Anschluß betragen die Grundgebühren beı cincr Übertragungsratc von $4.8 \mathrm{kbu}$ DM $310,-$ und be1 $48 \mathrm{kbit} / \mathrm{s}$ DM $2500,-$ monatlich. Dagegen betrage dic Grundgebühr für cinen ISDN-A nacliluß mit $2 \times 64 \mathrm{kbue} / \mathrm{s}$ nur DM 74,- S. zu dieser Pressgestaliung, durch dic dic Fernsprechkunden die ISDNEinlührung subventionteren, kntsch z. B. Peser Berger u.a., Optionen der Telekommunikzton, Düsseldarf 1988, II, S. 68 if., Solf., gs ff.

$4 \mathrm{~S}$, z. B. Bundesminisceriun für dis Post- und Ferninetdeweren, Das Fernmeldencex der Zukunit: ISDN, Bonn o. J.

s Bisher wurden für den Datenaustausch mur ctwa 2 55000 , für Telex 145000 und für Teletex an die 20000 Anschlüsse engerichtet - s. hierzu Mathieas Hisber, Universalität und Wirtschaftlichkeıt im Verbund, Computerwoche-Focus 4, vom 22.9.1989, S.6.

6 Vgl. I.F z. B. Herber Kuback/Amo Rolf, Mlikropolis. Hamburg 1986. S. 26 ff. und ausiührlich das 
ein einheitliches Telekommunikationsneť ermöglicht clcktronischen Zahlungsverkehr und überbecriebliche Rationalisicrungen in Handel und Banken. Die elekrronische Übertragung von Texıdokumenten soll den geschäftlichen Briefverkehr ersetzen und so Zeit und Kosten sparen. Anschlüsse in den Haushaleen schaffen die Voraussetzung dafür, daß die Kunden von zu Hause aus Waren fernbescellen, ihre Bankkonten verwalten, Auskünfte einholen, Reisen buchen und sogar ihre Arbeit erledigen können. Außerdem ermöglichr cin einheitliches Netz, neue Dienstleistungen, wie etwa den Zugang zu Literatur- und Infornacionsbanken, breit anzubieren.

Telekommunkationstechnik soll zum Straßennerz der Informationsgesellschafu werden. Um den wachsenden Bedürfnissen nach Telekommunikation nach- oder eher zuvorzukommen, wird die vorhandene Fernmeldeinfrastruktur in den nächsten Jahren und Jahrzehnten schrittweise grundlegend verändert. Die Dcutsche Bundesposı TELEKOM ${ }^{\prime}$ will in Übereinstimmung mit den Fernmeldeverwaltungen anderer Länder und europäischen Planungen ${ }^{8}$ das heucige Fernsprechnetz modernisicren. Ziel ist es, auf Basis des verlegten Kupferkabelnerzes (Fernsprechnetz und IDN) ein einziges Netz (ür alle Nachrichtenformen zu schaffen.

Weil alle heure bekannten Dienste mit Ausnahme der Bewegtbildübertragung über dieses Netz angeboten werden sollen, wird das künfrige Netz auch $»$ Integrated Services Digital Networke (ISDN) genannt. Die schrittweise Umstellung des Netzes hat bereits begonnen. Es soll 1993 flächondcckend angeboten werden und in spätestens 30 Jahren abgeschlossen sein. Dazu wird auf den vorhandenen Kabeln das Übcrtragungsverfahren gcändert, und es werden die hcurigen elektromechanischen Vermittlungseinrichtungen durch Prozeßrechner ersetzt. Die Kosten dieser Umstellung werden bis 1995 auf etrua 40 Milliarden Mark und insgesame bis 2020 auf etwa 140 Milliarden Mark veranschlagt.?

Diese Umstellung berrifft jedoch niche nur die öffentliche Telckommunikation. Sie erfaße auch die Nebenscellenanlagen innerhalb von Organisationen, Behörden und Unternchmen, die das öffentliche Telefornetz in den sprivaten. Bereich verlängern. Obwohi sie über eine ganze Stade verteilt sein und mehrere hundert oder gar tausende Endgeräte verbinden können ${ }^{10}$, wcrden sie nicht von TELEKOM, sondern von ihrem jeweiligen Inhaber berrieben. Sie sind für den Anschluß an das öffentliche

Zukunfesbilde in Alexander Koßßnagel/Peter W/dde/Volker Hanmer/Ulrach Pordesch, Dic Verieczlichkell der Inlormationsgesellschaft, Opladen $1989,18 \mathrm{ff}$.

7 Bundesministerium fur das Post- und Fernmeldewesen (Fn. 4 ); Xonzeption der Bundesregitrung zur Ncuordnung des Telekommunikatıonsmarkies, BT-DrS, $1 / 2855$; Bundesnunister lür Forschung und Technolog:t, Zukunftskonzept Informationstechnik, Bonn t989, S. 120 ff.

8 S. z. B. das Grünbuch der EG über die Entwicklung des Gemeinsamen Marktes fúr Telekommunkauonsdienstletstungen und Telekommunikatıonsgeräıc. BT-DrS 11/910; in den zuständigen europäischen und internationalen Gremien CEPT (Konferenz. der curopäischen Post- und Fernmeldeverwaltungen) und CCITT (Internacionaler beratender AusschuB für das Telegraphen- und Fernsprechwesen) wird sett emem Jahrchint sntensiv an der Erstellung internatoonaler Normen für ISDN gearbeitet. Um diese Arbert zu intenstvieren, wurde in März 1988 en Europäisches Instıtut für Telekommunikatıonsnormen gegründet. Zur Standardisterung s. auch Wolfgang Berndt, Dic Bedeutung der Standardisscrung im Telekommunikationsberesch für Innovation, Wettbewerb und Welehandel. Jahrbusts der Deutschen Bundespost 1986, S. $87 \mathrm{ff}$.

9 S. z.B. Der Bundesminister für das Post- und Fernmeldewesen, Miludfnsuges Programm für den Ausbau der technischen Konmunikationssysteme, Bonn 1986, S. 24: Helmut Schön, ISDN und Okonomie, Jahrbuch der Deutschen Bundesposi 1986, S. $16 \mathrm{f}$. Wenn dic TELEKOM-Kunden allerdings westerhin überwiegend nur telefonieren und die unaloge Anschlußletung behalten wollen, ist nuche auszuschließen, daß es nach dem "BTX-Flop" zu esnem $=15 D N-F l o p u$ komm - s. herzu z. B. Heudi Haug, Standard Ferug Los! ISDN - schon heutt mit Anwendernutxen?, Computermagazin 6/ 1988, S. 27; Mathbuts Huber (Fin. 5), S. 28; Herbert Kubucck, Das iniegrierte Fernmeldenetz der Bundespost - staatliche Investutionspolitik mit viclen Unbekannicn, Sozialwissenschafuliche Informawonen 1988 , S. 227 ff.

10 In der Bundesrepublik sind derzen erwa 13 Millionen Endgeralic an Nebcrsstellenanlagen angeschlossen -s. Matubias Huber (Fn. s) S.6. 
Telekommunikationsnecz konzipiert und müssen sich folglich mit dicsem weiterentwickeln. Daher werden derzeit fasc nur noch ISDN-fähige Nebensrellenanlagen inscalliert. Wcltweit werden derzeit jährlich Nebenstellenanlagen für last 20 Milliarden Mark verkauft. " Dieser Umsatz dürfte sich in den nächsten 10 bis zo Jahren, bıs nahezu alle Nebenstellenanlagen auf ISDN-Standard umgerüstet sind, noch deurlich erhöhen. ${ }^{22}$

Diese Planungen sind nicht ohne Kricik geblieben. Sie richtet sich vor allem auf die Folgen einer ausgeweiteten Telckommunikationsnutzung: Die derzeitigen Erkenntnisse lassen befürchten, daß sie die Rationalisierung beschleunigt und die Probleme der Massenarbeitslosigkeit verschärft' ${ }^{13}$, soziale Beziehungen zerstört und Vereinzelung versiärkt'4 sowie die Verletzlichkeit der Gesellschafı erhöht." Einige der vielfältigen rechulichen Implikationen solcher mirtelbaren Folgen einer Informatisierung der Gesellschaft, die durch die Modernisierung der Telekommunikation ermöglichı wird, wurden an anderer Stelle untersucht. ${ }^{16}$ Dic folgenden Ausführungen konzentrieren sich daher auf die rechtliche Bewertung einiger unmitcelbarer Auswirkungen der ISDN-Technik.

Was isc mun das für die rechtliche Beurteilung Neuc an ISDN?" Wesentlich für die ncuen Übertragungsverfabren ist der Üborgang von der analogen zur digitalen Übertragung. Bisher wurden die Schalischwingungen der Sprache über ein Mikrophon aufgenommen, in (analoge) elektrische Ströme verwandelt, die den Schallschwingungen ensprechen, und am anderen Ende der Lcitung über cinen Lautsprecher wicder in Schallschiwingungen zurückvenvandelt. ${ }^{8} \mathrm{Im}$ ISDN dagegen werden alle Arten von Nachrichten - Sprache, Bild, Text und Daten - in Form ciner Folge von binären Signalen ("Nullen und "Einsen ") übertragen. Sofern dic Nachrichten nicht wie zu übertragende Daten im Prinzip schon in dicser Form vorliegen, müssen sie in diese Form überführt (digitalisierc) werden. Für die Sprachübertragung etwa worden den Amplitudenwerten der Schwingungen feste Worte inncrhalb eines Rascers zugeordnec. Diese digitalen Werte werden in Folge übertragen und im

i) S. Zant $35 / 1989$ v. 29.8 .1959$. S. 25 .

12 Allen für Westeuespa wird bis zur Jahrausendivende mu Investutuonen von etws cencr Billion Mark gerechnet - s. Spregel $18 / 1989$, vom 18.9.1989, S.253.

13 S. z. B. Herbert Kubicek/Amo Rolf (Fn, 6), S. 2s\} ff., Volker Bahl, Informationsgesellschaft als Fluch vor dem Arbetrsrecht, in: Alexander RoBasgel (Hrsg.). Freihert im Griff, Informationsgesellschaft und Grundgesel\%, Siungart 1989, $107 \mathrm{ff}$.

14 S. z. B. Herber Kubscek/Amo Rolf (Fn, 6) S. $2386 f$; Barbara Menler-Meibom, Soziale Kosten in der Informatsonsgesellschaft, Frankfurt 1987: Wibalm Stemmidler ( $\mathrm{H}_{5 \mathrm{sg}}$.), Verdatel und vernect, Franklur 1989

is S. hierzu Alexander Roßnugel n.a. (Fn.6) und Ulrob, Pordescb. Zum Katastrophenpotenual der Telekommunikatıon, Zivilvertendigung $\mathrm{II} / 1989$. S. 4 iff.

i6 S. hrerzu ausfuhrlich Alcxander Roßnagel/Peter Wedde/Volker Hammer/Ulruch Pordesch, Digualisıenong der Gundrechte? Zur Verlassungsvertraglichket der Informations- und Kommunikationstechnik. Opladen 1990 sowe Alexander Roßnagel (Hrsg.). Freihest um Griff, Informauansgesellschait und Grundgesetx, Suutgan 1989 .

17 Im folgenden werden nur die wicheigsten Grundzüge dargestells. Zur tcchnıschen Konzeption von ISDN s, näher Karl Hemz Rosenbrock. ISDN - ene folgenrichuge Weiterentwicklung des digitalen Fernsprechnetzes, Jzhrbuch der Deutschen Bundespost 1984, S. 509 ff., Helnut Scloön, Die Deutsche Bundespost auf ihrem Weg zum ISDN, ZPF 6/1984, S. 20 ff., Peter Bocker. ISDN - Das dienstintegricrende digitale Nachnehennetz, 2. Aufl. Berlin u. a. 198\%: Albrecis Albensoder (Hrsg.). Telekommunikation - Netze und Dienste der Deutschen Bundespost, Heidelberg 1987: Herbert Kribicek/Arno Rolf (Fn. 6); Peler Berger (Fa. 3) S. 1/8g ff. In folgenden wird eine sdealiypische Unterscliendung zwischen der ISDN-Technik und der bisherigen Fernsprechtechnik getroffen. Die realexistserende Kommunikatonsechnik Ist jedoch enne Technik im Übergang, die derzeit alle nöglichan 7.wischenformen auf weist.

18 Berets sett 1979 werden die Übertragung und die Vermielung im Fernspreclanctx nach und nacls suí digitale Signale umgestellt. Die Wenerentwicklung dieses digitalen Fernsprechnetzes zu ISDN mis den im folgenden beschricbenen Merkmalen erfolgt allerdings erst sen ig 9 - s. hierzu z. B. Orıo Hilz/Haris Klem, Einsatzstrategue für digitale Vermutlungstechnik im Fernsprechnetz der Deutschen Bundespost, Jahrbuch der Deutsclien Bundespost 1984, S. 51 ff., Kayl Heinz Rosenbrock (Fn. 17), S. jog ff., Helimut Schön (Fn. g), S.gif. 
gegenüberliegenden Endgeräı gegebenenlalls zurückverwandelt. Die Übertragungsrate im ISDN ist hoch genug, um über dieselbe Leitung zwci Übertragungskanäle und cinen Steuerungskanal zu vermitreln's und daher gleichzeitig zwei Endgeräre zu betreiben. So wird es möglich sein, über einen Hauptanschluß gleichzeitig zu telefonieren und dem Gesprächspartner eine Zeichnung fernzukopieren. Insgesamt sollen bis zu achr Endgeräre anschließbar scin, die die Nutzung aller heute angebocenen Dienstc zur Daten- und Textübertragung ermöglichen.

Neben der Digitalisicrung der Übertragungsverfahren ist die speicherprogrammierbare Vermutlungstechnik die zweite wescntliche technische Änderung. Bisher ist dic Technik der Fernsprechvermittlung noch übcrwiegend elektromechanisch. Jeder Ziffer, dic über die Wählscheibe oder -tastatur eingegeben wird, seczt in der jeweils zuständigen Vermittungsstelle auf dern Weg zum gerufenen Teilnehmer jewcils einen kleinen Elektromotor in Bewegung, der die Kontakre auf die richrige Stelle drehr und so dic Verbindung weiter durchschaleer. Dadurch werden nirgendwo im Netz die Verbindungsdaten Festgebalien. ${ }^{20}$ In rechnergesteuerten Vermirtungssystemen, wie sie in den Datennetzen heute schon eingesetzt werden, übernehmen Programme die Herstellung von Verbindungen. Dabei werden die Wahlinformationen des Teilnehmers nicht mehr schrittweise umgesetzt, sondern sie sind in ihrer Gesamtheit Eingabedaten für die Rechnersteuerung, dic als Ganzes ausgerverter werden. Aus diesem Grund ist es technisch notwendig, die Verbindungsdaten zumindest für die Dauer der Verbindung zu speichern. ${ }^{2 \prime}$

Die hohen Übertragungsraten und die Flexibilitäc von rechnergesteuerten Vermirtlungseinrichtungen ermöglichen es künftig, dem Teilnehmer zusätzliche, den Kommunikationskomfort steigernde Dienstmerkmale anzubieten, wie etwa das Umleiten, Weitcrleiten, Nachziehen, Heranholen und Übernehmen von Anrufen, Konferenzschaltungen, Rundruf, Teamfunktionen, die Einrichtung geschlossener Benutzergruppc, das Aufschalten auf Verbindungen, Kurzwahl, Direktruf, Wahlwiederholung, Makcln, Freisprechen, Lauthören, die Anzeige des rufenden Teilnehmers, das Anklopen bei einem besetzten Teilnehmer, sutomatischer Rückrul im Frejund Besetztfall, Sprachspcicher und viele weitere mehr."

Telekommunikation soll wie Strom aus der "Universalsteckdosen verfügbar werden. Mit dem Ausbau der cinheitlichen Infrastruktur soll sich die Telckommunikation immer mehr auf das ISDN verlagern, bis die Nuzzung der herkömmlichen Fernmeldenerze entbehrlich wird." Mit dem ISDN wird ein Netz verfügbar, das Endscellen in jedem Haushalt und jeder Organisation haben kann, womit sich ein

19 Zwei Nutzkanale mie jeweils $\sigma_{\$} \mathrm{kbu} / \mathrm{s}$ und en Zuchonkanal mit i $6 \mathrm{kbiu} / \mathrm{s}$. Damit ist es auch schneller

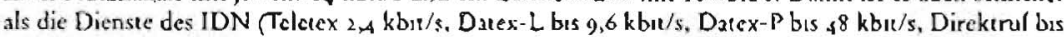
$48 \mathrm{kbu} / \mathrm{s}$ ) - s. hierzu z. B. Kurl Hesnz Rosembrock (Fn. 17), S. s12 f. Durch dic hohen Übertragungsraten dauer die Übermulung eıner DIN-A-4-Sete mu Telesex stal busher 8 Sekunden im IDN nur noch weniger als ene Sekunde und mit Telefax statt bisher i bis 6 Minuten im Femsprechnet? nur noch s bis 10 Sekunden - s. Mattbas Huber (Fn. s), S. T.

$20 \mathrm{Um}$ sic im Rahmen einer Vergleichszahlung zur Gebuhrenkonirolle $\left(\$ 8_{4}\right.$ Abs. 1 Nr. S TKO) oder zur Feststellung ankommender Wählverbindungen $\left(\$ 8_{4}\right.$ Abs. 1 Nr.6 TKO) dennoch zu erlangen, missen besondere Vorrichtungen an der Anschlublettung des Teilnehmers eigens angebrache werden.

21 S. Bundesregitrung BT-DrS $11 / 28,3,5.4$; s. hierzu allerdings den Alternauvvorschlag in Herbert Kubucele/Arno Rolf (Fn. 6), S. 32 fff., Peter Berger w. a. (Fn. 3): S. II1/44ff.; Herbert Kubicek, Entwickiungspiade der Telekommunikation, in: Alexander Roßnagel (Hrsg.), Freihert im Griff, 1989, S. Is r ff.: cınt weitere Technikalternatıve zum Schutz der Kommunikationsdaten cnthale Andreas Pfitzmann/ Birgul Pfrizmam/Michacl Wasdner. Datenschurz garanuerende offenc Kommunikationsnerze, Intor* matik-Spckurum 1988, S. $118 \mathrm{ft}$.

2. S. Lierzu ausführlich Volker HammuriUlnach Pordesch/Alexander Roßnagd, Gicstalungsanforderun. gen fur dic ISDN-Nebensicllenanlage der Hochscluulregion Darmstade, Provet-Berscht 3, Darmstad. 1989 , S. 41 ff.

23 Um auch höhere Datenübertracungsraten als 64 kbit zu erzıelen, sollen ab ; 990 parallel zur Einführung des ISDN Zug um Zug Glasisserkabel zu Teilnchmeranschlüssen verlegt und dazu Brettbandvermutlungseınrıchtungen (zunächst als Ergänzungstechnik) installiert verden, die Úbertragungsgeschwindig- 
breiter Anwenderkreis ergibt. Hier schafft ISDN mit seinen standardisicrten Sclunittstellen und einheitlichen Leistungsmerkmalen beste Vorausscizungen für die sogenarnte xoffene Kommunikation* und für eine gewalcige Ausweitung der Telekommunikarionsanwendungen.

ISDN wirbt mit vielen Erleichterungen und Verbesserungen für den Austausch von Nachrichten. Mit diesen Vortcilen sind aber auch jeweils spezifische Risiken verbunden. Im Vergleich zur bisherigen Fernmeldetechnik verursachen insbesonderc vier Merkınale der ISDN-Technik neue Risiken für Freiheitsrechrc:

- Die Digitalisierung aller Informationstypen ermöglicht die Integration aller schmalbandigen Dienste in cinem Nerz mir einlyeithcher Vermitrlungstechnik. Die Anlage vermittelı nicht mehr Telefongespräche, sondern Datenübcrtragungskanäle z wischen verschiedenen Anschlüssen, über die je nach Endgcrät Sprache, Text. Bilder und Daten ausgetauscht werden können. Zwischen allen Diensten kann freizügig gewählt und ihre Leistungen können kombiniert werden. Die Multifunktionalität von ISDN erschließt vielfältrige Anwendungsmöglichkciten der Rechnerkopplung und Bürokommunikation. Sie eröffnet aber auch ncue Risitsen der Rationalisierung, Kontrolle und Datenspeicherung.

- ISDN arbeiter mit einer sof twaregesteuerten Vermutulungstechnik. Scin Leistungsumfang ist daher nur bis zu einem gewissen Grad festgelegt, er kann durch Einspielen neuer Sofrware verändert werden, Dem Vorteil, daß die Anlage Mexibel veränderten Anfordcrungen angepaßt wcrden kann, steht jedoch das Risiko gegenüber, daß Veränderungen unkonırolliert und mißbräuchlich vorgenommen werden können. Außerdem werden - tcclınisch bedingr - wenigsiens kurzfrisrig Verbindungsdaten gespeichert, dic durch weitere Programme - etwa zur Gebührenabrechnung - ausgewerter werden können. Dem Vorteil der höheren Ausgabentransparenz einer detaillierten Gebührenabrechnung stehı das Risiko gegenüber, daß diese Daten zur Bildung von Kommunikationsprofilen mißbraucht werden.

- ISDN bictct die Möglichkeit, Nachrtchtenunhalte zum Zwecke zeitversetzter Nachrichtenübermitiung auf internen oder externen Speichern abzulegen, damit sie von anderen Teilnchmern abgerufen werden kämnen. Dem Vorreil, Kommunikation zeitlich zu entkoppeln, steht hier das Risiko gegenuber, daß Dritte auf dic Inhalte der gespeicherten Nachrichten zugreifen.

- ISDN bieter standardmäßig erheblich mehr und komplexere Leustungsmerkmale als dic heute überwiegend noch eingeseczten Anlagen mir Analogtechnik. Dieser Vortcil ist jedoch mic dem Risiko verbunden, daß der Zustand des Systems für die Nutzer intransparent wird, dic Autonomie der Arbeitsgestaltung und die Selbstbestimmtheit der Kommunikation eingeschränkt sowic die Anwesenheit und Arbeisleistung unbemerkt kontrollicrt werden.

\section{Risikoadäquate Rechtskonkretisierung}

Kommunikation isc das Mcdiun der Pcrsönlichkeitsbildung. Ydenticät entsteht nur in Kommunikation, Individualizät wird nur in der durch Kommunikation vermitecltcn Erfahrung des Andersseins gebildet. Selbständiges Lernen und selbsrbestimmtes Herausbilden und Aneignen eigener Wertc und Beurteilungskriterien sctzcn Kom- 
munikation voraus. ${ }^{24}$ Information, Meinungsfrciheit, Wissen und Kunst gründen auf Kommunikation. Kollektuve Interessenvertretung, Versammlungs-, Vereinigungs- und Koalitionsfreibeit setzen Kommunikation voraus. Politische Willensbildung und die Organisazion politischer Interessen erfordcrn eine freic Kommunikation ebenso wie wirtschafłliche und berufliche Bctätigung, privater und familiärer Austausch. Gęsellschaftliche Integration, demokratische Willensbildung und staatliche Wirkungseinheit sind ohne Kommunikation nicht möglich.

AuBerhalb der Rufwcite ist Kommunikation auf technische Untersiüzung angewiesen. Aut gesellschaftlicher Ebene sctzt sie ein technisches und organisatorisches System der Kommunikationsunterscützung voraus - zucrst zeitlich versetzrc mündliche Übertragung durch Boten, dann Schriftverkehr durch Postdienste, später kurafristige schriftliche Übertragung durch Telegraphe und schließlich das aktucll gesprochene Wort durch Telefon, zcitgleiche Übertragung von Daten, Text und Bilder durch die Telematik. Sobald cin Telekommunikationssystem funktioniert, stellen sich die Menschen in ihren Arbeirs- und Lebensgewohnheiten darauf ein und werden in ihrer Kommunikarion von ihnen abhängig. ${ }^{25}$

Moderne Arbeirsmethoden und Lebensgewohnheiten sind auf das Telefon, of auch schon auf dic Datenübertragung angewiesen. ${ }^{36}$ Eine Veränderung der Telekommunikationstechnik wirkt sich daher durchschlagend auf die Lebens- und Arbeitswelt aus. Sie ist nicht nur ein neutrales Medium zur freien Gestaltung der Kommunikation. Sie legt vielmehr dic Strukturen fest, innerhalb deren der Einzelne agieren kann. Telemariksysteme erbringen akcive Lcistungen und zwingen - je komfortabler sie sind, um so mehr - den Nutzern ihre Bedingungen auf. ${ }^{27}$ Künfitig ist davon aber nicht nur das akcuell gesprochene Wort betroffen. Die qualitative Ausweitung der Telekommunikation erlaßr auch alle anderen Kommunikationsformen - die Speicherung des gesprochenen Wortes, dic Überragung von Bildem (Kopicn, langsames Bewegtbild, Bildfernsprechen), von Texten (Telebriefe, clektronisch publizierte Bücher, Datenbankauskünfte) und von Daten. Wenn künfrig auch - wic cs erwartet wird - zumindest der schriftliche Geschäftsverkehr oder ein gewisser Teil des privaten Schriftverkehrs über Telefax, Teletex und Bcx abgewickelt wird, dann ist jede Entaußerung über die Rufweite hinaus durch Telematik vermirtelt.

Telekommunikation ist Voraussetzung für die Wahrnchmung nahezu aller Grundrechre. Technik erweiter jedoch nicht nur die räumlichen und zcitlichen Möglichkeiten von Kommunikation und Information, sondern serze diese auch neuen Begrenzungen, Zwängen und Gefährdungen aus. Ihre Nutzung führt zu einem Konflikt zwischen der Unterstützung des einzelnen bei der Wahrnehmung seiner Selbstbescimmungschancen und der Bevormundung seiner Akrivitäten. ${ }^{28}$ Die spezifische Technik der Kommunikationsvermitrlung und Informationsverarbcitung ist daher grundrechtsrelevant. Die soziale und personale Basisfunkrion von Kommunikation und Information für dic Grundrechrsausübung muß daher selbst unier den

24 S. hierzu z. B. Adalbert Podlech, GG-AK Arr. I Rdnr. 39 und Are. I Rdnr. 13, 18; Niklas Lubmaun, Grundrechec als Institution, Berlin 1965 , S.61ff., 66ff., 72; Dieter Subr, Entfaltung dur Micnschen durch die Menschen, Berlin 1976, S.80ff., S8I. m.w.N.

is S. hierzu z. B. Klaus Beck, Telefongeschuchie als Soxialgeschichee: Die sozialc und kulturdllc Aneignung des Telefons im Alliag, in: Forschungsgruppe Telctonkommunikation (Hrsg.), Telclon und Gesellschatt, Berhn 1989, S. ss ff; Uinch Lange, Telefon und Gescllschaft, ebenda S. 27 if; ders., Menkommunikation schalfit neue Konventionen, Computerwache-Focus 4 vom 22.9.1989. S. 8 fl.

26 S. 2. B. Ulrich Pordesch (Fn. 15). S. 4:.

27 S. bxeru Hans Brindemam, Rechliche und poliessche Kontrolle eincr neuen Infrastrukeur, in: Gescllschali fur Rechiss- und Verwaltungsinformatik (Hrsg.). Konmunikationstechnssche Vemeizung. Rachisprobleme, Kontrollchencen, Kliconcrinteressen. Darmstade 19\$6, S. 25 ff.; Alexander Roßnagel 11. 4. (Fn, 16), S. 43 f.

28 S. z. B. Spuros Simitis, Selbsibestummung: [llusorisches Projeke oder reale Chance, K] 1988, S. 43. 
Schurc der Grundrechte fallen. Verändert sich das technische und organisatorische System der Telekommunikation und verursacht diese Veränderung neue Risiken, so müssen auch die Grundreche neu konkretisiert werden, um die Scluutzziele unter verändertcn Bedingungen ohnc Einbußen gewährleisten zu können."

Die Sichcrung der Selbstbestimmung des cinzelnen erfordert im Zeitalter des ISDN eine Konkretisierung der Freiheirsrechte, die den neuartigen Gefährdungen gerecht rvird. Soweit es der Schuť der Persönlichkeit gegen neve Risiken der Technik erfordert, darf sich »das Recht... in diesem Punkı der tcchnischen Ennvicklung nicht beugen $4 .^{\circ}$ Dabei darf es nicht genügen, organisacorische Regclungen und Verhaltensanwcisungen an die Menschen zu fordern. Vielmehr iss Sicherheic und Gewißheit menschlichen Verhaltens vor allem durch entsprechende Gestaltung der Technik «u gewährleisten - also durch Anforderungen an die Technik als verfestigtes menschliches Handeln. Dagegen sind rechtliche Regeln für die Nutzung der Technik, die allein das Verhalten der Menschen den Erfordernissen einer als gegeben hingenominenen Technik anpassen, unzurcichend."' Sozial unerträglicher Gebrauch der Technik muß nicht mehr verboten und kontrolliert werden, wenn er durch sozialverträgliche Gestaltung technischer Systeme ausgeschlossen ist. Entsclieidend ist also, ob aus Grundrechten solche tcchnische Gestaltungsanforderungen abgeleiex werden können. Dabei komme es darauf an, die Gefährdungen zu vermeiden, ohne die Vorteile für dic Ausübung von Grundrecheen zu verlieren. Lediglich als ¿weitbeste Lösung wäre zu erwägen, Risiken durch Verhaltensanforderungen cinzuschränken.

Die Vertraulichkeit individueller Kommunikation, soweit sie fernmeldetechnisch übertragen wird, ist das Schurzgut des in Art. 10 Abs. 1 GG gevahrleisteten Fernmeldegeheimnisses. Dieses Grundrecht gewährleistec dic frcie Entfaltung der Persönlichkeit durch einen privaten, vor den Augen der Öfentlichkeit verborgenen Austausch von Nachrichten, Gedanken und Meinungen ${ }^{32}$. Ausreichender Schutz gegen die Risiken der ISDN-Technik ist also in erster Linie in einer Konkrecisierung des Fernmeldegehermnusses zu suchen. Denn cs schützr nicht nur die Vercraulichkeit des Inhalts, sondern auch des $\mathrm{Ob}$ und des Wie der Kommunikation", und erstreck sich auf alle Formen der Nachrichtenübertragung, die mit Mitreln des Fernmeldeverkehrs stattfinden - also auch auf alle neuen Formen individueller Telekommunikasion. ${ }^{4}$ Art. ro Abs, 1 GG ist also einschlägige Schutznorm, sowcit Telekomnuni-

29 S. x. B. Adalbert Podlech. GG-AK, Ari.z I Rdnr. 54; ob dic Grundrechte als Abwchrnecht, Schutzpllichı oder Teilhaberecht zu konkretusieren sind, hangı von de. Umsianden ab. unter denen tarsächljche Grundrechisausubung ermoglicht werden kann -5 , herzu unten 6.

30 BVerfGE 35, 202 (227) zitiert hier BGH NJW/1966, S. 2154.

31 S. z. B. Jorebim Scherer, Rechesprobleme des Datenschulzes ber den "Neuen Medien», Disseldorf 19\$9. S. 2 l.: Günter Dürg. in: Maunz/Dung, GG-Kommentar, Art. 10 Rdnr.21: Hans-Joaclinrt Kerkau, Neuc Medien: Neue Risiken, neues Recht?. n; Der Berliner Dacenschutzbeauftragic (Hrog-), Ditenschutz und Neve Medien, Berlin 1987, S. 101., Spiros Simats (Fn. 28), S. 37 f; Alexander

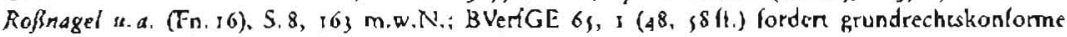
Vorzusseczungen fur die Verarbelung personenberogener Daten.

32 BVeriGE $67,157(171)$

33 BVerfGE 67, 157(172); s. herzu auch $₫$ ro Abs. I S. 3 J:AG sow/c x. B. VG Bremen. NJW 1978, S.67: ingo e. Mïuch. Uncilsanmerkung. NJW 1978, S. 67: C̈̈̈ner D̈̈rag in: Maunz/Durg, GG-Kanmentar, Ar. 10 Rdnr. 18; Hans-Uwe Erichsen, Festsiellungsklage und Innenreche, Grundrechic und Amisverwaliung, VerwA 1980, S. 436; Erich Pappermann, in: I. v. Munch, GG-Kommentar, 3. Aul, Art. 10 Rdnr. 18; Wolfgang Schatzschneuder, Fernmeldegeheimnis und Tclefonbeschattung, NJW 1981. S. 268; Peter Badura. Dic Tragiveite des Rechts auf informationclle Selbsebestımmung für die nornative Regelung der öffentichen Telekommunikauonsdienste der Deutschen Bundespost, Jahrbuch der Deutschen Bundesposi 1989, S. 20 f.; Hansjürgen Garstka, Datenschuzz in Telckommunikationssystc mon, in: Valk, R. (Hrag.), Gl-18. Jahrestagung, Berlin u. a. 1988, S. 666.

34 S. z. B. Bodo Pierolh/Bernhard Scblink, Grundrechte, 3. Auíl. Headelberg 1987, Ronr. 861; Joachum Scherer (Fn.31) S. 24 . 
kation dem Risiko der Einsichtnalıme staatlicher Stellen ausgesctzt ist." Er gewährleistet jedoch keincn umfassenden Schutz gegen alle Risiken, dic von den neuen Telekommunikationstechniken für Freiheitsrechte ausgehen können. Denn er gewährleister zum Beispiel nichı die Transparenz der Kommunikacionsvorgänge oder den Entschcidungsspielraum der Kommunikacionspartner ${ }^{36}$

Das Fernmeldegeheimnis schützt lediglich einen speziellen Ausschnitt der Persönlichkeit - nämlich vor Kennenisnahme des durch Kommunikationssysteme uncerscützten Austausches von Informationen. ${ }^{17}$ Soweit scin Schutzbereich reicht, grelit cs Arr. 2 Abs. I GG vor. ${ }^{8}$ Jenseits dicses Schutzbereichs ist aber cin umfassender Schurz gegen die verbleibenden Freiheitsrisiken des ISDN nur in einer risikoadäquaten Konkretisierung des Grundrechts aus Art. 2 Abs. I GG zu gewinnen.

Das durch Art. 2 Abs. 1 gewährleistete sRecht auf frete Entfaltung der Persönlichkeıt schützı zwei A usprägungen menschlichen Seins: das Handeln oder Unterlassen, das die Freiheit seiner Entfaltung begründet, ${ }^{39}$ und - in Verbindung mit dem Schutz der Menschenwürde durch Art. 1 Abs.. GG - dic Verhaltenswcisen, die seine Pcrsönlichkeit konstituieren.40

Was aber ist - im Hinblick auf ISDN - unter Entfaltung und Schutz der Persönlichkeit zu verscehen? Allein aufgrund der sprachlich offenen Fassung der Grundrechte läßt sich nicht mit Eindeutigkcit entscheiden, ob beispielsweise der aucomatische Rückruf die Entfaltungsfreihcil des Angerufenen oder dic Speicherung von Gebührendaten das Persönlichkeitsrecht des Rufers becinträchrigen. Eine Interprecation im Sinn der Erkenntnis eines vorgegebenen Inhalts ist wegen der Weite und Offenheit der Sprachstruktur der Grundrechte nicht möglich. Was unter die Begriffe im konkrecen Fall gefaßr werden soll, is nicht ohne Analyse der Rcalicät zu entscheiden, die die betreffende Nom zu ordnen bestimmt ist. Da die zu regelnde Wirkliclakeit - vor allem auch durch technische Innovarionen"' - geschichtlichen Veränderungen unterliegt, ändern sich mit ilar die Ergebnisse der Normkonkrerisierung - obwohl der Normtext identiscli bleibr."12

Die Grundrechte auf Entfaltungsfreiheit und Persönlichkeirsschutz sind für die Technikbewerrung aber nicht nur wegen der Weite ihres Schuczbereichs, sondern vor allem wegen ihrer Funktion bedcursam, auch gegen nneue Gefährdungen der menschlichen Persönlichkeit đ durch die moderne Entwicklung der Technik Schutz zu gewähren."13 Um dieses Schurzziel zu erreichen, erfordern sic immer wieder zeicgemäße und gefährdungsadäquate Konkretisierungen ihres Schuzzgehalts. ¿t Um

3s Entsprechend den technuschen Möglichketen wurden im Vorgriff auf ISDN die Kontrollbefugnusse des Verfassungsschutzes, des Bundesnachrochtendienstes und des Militärischen Absclirmdienstes aul alle Telekommunikatıonsdienstc, auch auf alle - auch privacen - Anbieter von Telekommunikalıonsdiensicn in den durch das Poststrukturgeselz neugclabien $\$ 1$ des Gescizes zur Beschránkung des Bricf-, Post- und Fernmeldegehemunsses dusgeweitet.

36 Ein wetere's Defize enthäle Art. 10 Abs. I GG, wenn er ausschließlich staxtsgenchea interpretuen wird und ihm keune milelbarc Drietwirkung zucrkanne wurd - 5. hierzu z. B. Cbrestoph Gusy, Das Grundrecht des Post- und Fernmeldegchamoisses, Jus 1986, S. 92, 95 .

37 S. hierzu z. B. BVeriGE 67, 137 (169fl.); Chrsstoph Gusy (Fn. 36). S. 89lf.; Pcrer Badura (Fn. 33), S. 20 I.: Jaachim Schercr ([n. 31), S.21, 2s.

$38 \mathrm{BVerGE} 67,157(171)$.

19 S. z.B. BV 5 तGE 6, 32 $(37) ; 63,45(60)$

to S, x.B. BVerfGE, $4,14^{8}(153) ; 65,1(41) ; 72,135(170)$.

1I S. 2.B. BV GrfGE $57,295(318,322)$.

t2 S. z. B. BVeffGE 40,296 (315): $\{6,54$ (76 (1.): 73, 118 (154): 74, 297 (350) sowe Alexander Roßmagd, Dic Andenangen des Grundgesecees, Frankfure 19\$1, S. 27 ff., 8 off, und ders., Radioskciver Zerlall der Grundrechie, München 1984, S. 18 ff, jeweils m. w. N., speziell für An. 2 Abs.1 s. Adalbert Podlech, GG-AK, An. 21 Rdnr-17.

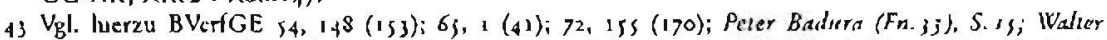
Schmill Glacser, Schuez, der Privatsphärc, in: Joseph Isensee/Paul Kirchhof (Hrsg-). Handbuch des Suatsreches, Band IV, S. S4

44 S. hictzu Adalben Podlech, GG-AK. Ar. 2 I Rdnr. ze, 47, 44 ; fur das Verhalins von Persónlichkeis- 
das Schurzziel beider Grundrechte auch gegenüber der neuen Techrik durchzusetzen, kommt es daher darauf an, sic im Hinblick auf die speziellen Risiken und das besondcre Anwendungsumfeld zu konkretisieren.

Eine solche Reaktion auf die Herausforderungen der Informatisicrung der Gescllschaft ist die Konkretisierung des Rechts auf freie Entfaltung und des Persönlichkeirsschurzes zu eincm Rechi auf informactonelle Selbstbestımmung. " Dieses "Grundrecht gewährleisret die Befugnis des Einzelnen, grundsäızlich selbst über die Preisgabc und Verwendung seiner persönlichen Daten zu bessimmen «. Beschränkungen dieses Reches sind nur nim überwiegenden Allgemeininteresse zulässig und dürfen nur sowcit reichen, wals es zum Schutz öffentlicher Inceressen unerläßlich ist *. Sie bedürfen einer ngeserzlichen Grundlage, aus der sich die Voraussetzungen und der Umfang der Beschränkungen klar und für den Bürger erkennbar ergeben * und in der "organisatorische und verfahrensrechtiche Vorkebrungena getroffen sind, wclche der Gefahr einer Verletzung des Persönlichkeitsrechts entgegenwirken ${ }^{46}$ Werden personenbezogene Daten unfreiwillig erhoben, serzt dies voraus, "daß der Geserzgeber den Verwendungszweck bereichsspezifisch und präzisc bescimm und daß die Angaben für diesen Zweck gecignet und crforderlich sind $\kappa^{.}{ }^{+}$?

Soweit im ISDN Daten verarbeitet werden, gewährleistet diese Grundrechtskonkretisierung auch'gegen Risiken der Telematik Schurz und ermöglicht, verfassungsrechtliche Maßstäbe für eine Bewertung des ISDN zu gewinnen. Zwei Beispiele:

ISDN-Anlagen ermöglichen die Anruferidenufizıerung, d.h. das Vermitslungssystem zcige dem Gerufenen auf einem Display die Nummer des Rufers an. Da für diesen Eingriff in das Recht auf informationelle Selbstbestimmung eine bereichsspezifische geseraliche Grundlage fehlt, ist sic nur zulässig, wenn der Anrufer damit einverstanden isc. ${ }^{8}$ Dic erforderliche Zustimmung zur Übermitrlung der Rufernummer könnre auf drcierlei Weise erteilt werden: Die optimale Lösung bestünde darin, daß der Rufer durch Tastenơruck an seinem Endgerät entscheiden kann, ob seine Nummer am Gerät des Gerufenen angezcigt wird.t9 Als zweitbeste Lösung wäre anzusehen, daß der Beginn des Identifikationsvorgangs dem Rufer durch die Vermittlungsstelle zuverlässig (für Geräte ohne Display akustisch) signalisiert wird. In der dazwischenliegenden Pause vor der Anzeige der Rufernummer besteh dic Möglichkeit, die Verbindung abzubrechen. Ebenfalls zulässig, den sozinlen Bedürfnissen allerdings am wenigsten gerecht werdend ${ }^{\circ}$, dürfre die Lösung sein, dic $\$$ ros

recht und informationeller Selbstbestummung so auch Enst Banda, Das Reche auf informatsonclle Selbstbestmmung und die Rechtsprechung des BVerfG zum Ditenschuly. DuD 19S A, S.89.

4s Das Persönlichkeıtsreche wurde dadurch nauf einen neuen Gefahndungasachverhall hin konkrctisicr * Emst Benda, Datenschutx und Grundgesetz, Siemens-Zestschrift i $8\left(198_{4}\right)$, S. S.

46 BVerfGE $65,1(436)$.

47 BVtrfGE 65, $1(46)$.

48 Ebenso der Landesbeauftragte für den Datenschuzz der Fresen Hansestadt Bremen, Einsatz von ISDNFähıgen Telefonnebenstellenanlagen in der bremschen Verwaltung, Bremen ig\$9, S. 10 f.; ders., 11. Täigkeitsbericht. Bremen 1989, S.46; Herbert Kubecek, ISDN im Lichec von Demokratiepnnz.ıp und informatsoncller Selbstbestummung, DuD 1987, S. 24 f.; Hans-Peter Holler, Datenschutz und Le1stungsmerkmale beı ISDN-Anlagen und -Endgeräten, SoTech-IVerkstattberiche Nr. 48 . Düsscldorí

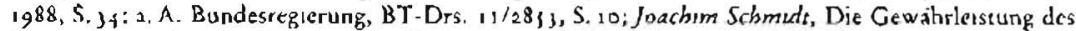
Datenschutyes beı der Teilnahme an Telekommunikationsdiensten der Deutschen Bundespost. Jahsbuch der Deueschen Bundespost 1988, S. 344 f. Der Hinwers von Hans Gliss/Geong Wronka, Dacen. schucz in Hicom, Sicmens PN PPA 4, München 19\$7, S. 13, der Anrufer gebe durch die Anzeeige nichi

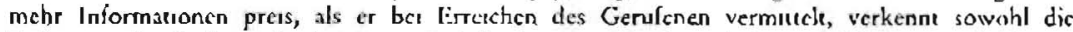
Bedeutung des Reches auf informationellle Selbsibestummung als such die tatsächlichen Möglichkesten Dniscr (ctwa bei Anwesenlieit ain Apparat des Gerufenen oder infolge cince Umleitung), von der Anz:age Kenntors zu nehmen.

49 Zur wejteren Optumierung dieser Lüsung durch en Shakehand-Verfahren s. unten s.

so Dies wird inzu'ischen auch in Teilen der Tclekommunikatıonsmduserie so gesehen. S. x. B. Dieter Klkmpp, Trchnikfolgenabschaizung: Bedingungen und Perspektıven in der kommunikationstichni- 
TKO vorsieht. Danach kann der Teilnchmer zwar nichı lür die jeweilige Verbindung, aber immerhin generell für alle Kommunikationsvorgänge beantragen, daß seine Nummer unterdruickt wird. Eine Identifizierung ohne Wahlmöglichkeit oder Signalisierung, wie sie in vielen Nebensrellenanlagen stattindet, ist jedoch unzulässig.

Das zweire Beispiel ist die Verarbetung der Gebitbrendaten durch TELEKOM bzw. die Bctreiber von Nebenstellenanlagen. Da die Daten, die nach dem ISDNKonzept technisch notwendig zwischengespeichert werden müssen, für die Gebührenabrechnung genutzt werden, kann im Gegensatz zu herkömmlichen Anlagen die Gebührenabrechnung eine Liste aller geführten Gespräche mit Datun, Uhrzeit, Koscen, Nebenstelle und Zielnumner beinhalten. Dadurch können die Kostentransparenz erhöht und inögliche Mißbräuche aufgcdeckt werden. Solche Einzclgebührennachweise legen jedoch auch Kommunikacionsbeziehungen offen und ermöglichen die Bildung von "Kommunikationsprofilen ${ }^{3 t}$

Für diesen Eingriff in das Reche auf informationelle Selbstbesrimmung der Gerufenen fehlt ebenfalls die bercichsspezifische gesetzliche Grundlage. Auch ist eine vollständige Er\{assung aller Verbindungsdaten - wie die bisherige Praxis der Deutschen Bundespose eindrucksvoll belegr - für den Zweck der Gebührenabrechnung nicht eríorderlich..'2 Hierfür genügt es, nur cinen Teil der Zielnummer zu speichern, so daß der Einzelgebührennachweis nur für den iarsächlichen Benuczer sinnvoll ist. ${ }^{53}$ Daß der Nachweis nur auf Antrag des TELEKOM-Kunden (gcbührenpflichtig) ausgestellt wird, ändern an dieser Bewcrtung nichts. Denn mit seinem Antrag kann der Kunde nur in die Beeintröchtigung seincr Grundrechee, niche aber in die der von ihm Angerufenen einwilligen." Ohne Zustimmung des Angerufenen ist eine vollständige Erfassung aller Verbindungsdaten recheswidrig."

Das Recht auf informationelle Selbstbestimmung schützt allerdings nur gegen gan\% spezifische Risiken neuer Kommunikationssysteme: Es greift nämlich nach herr-

schen Industne (Manusknpt), Stutgart 1989, S. 10, der die bisher fehlende Realisıerung der Identifizıerungstaste als groben Schnizzer bezenchnet und auf en svöllig schefes Anwenderbild einer ganzen Branche* zuruckführt, die sich bisher zuwenig um die Erkenntnısse der Kommunikatsonssozrologic und -psycliologie gekümmert habe. S. hierza auch Wemer Zucker, "Prvacyo suf schmalem Grat, Computcrwoche-Focus 4 vom 22.9.1989, 5. 14; Horst H. Ablke, Telekommunikationsordnung und Datenschurz, DuD $1987, S_{3} 84$ sowe unesn bet Fin. 91 .

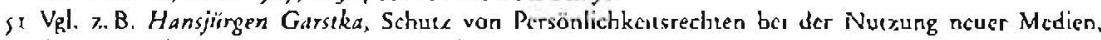
DVR-Beihcli 16, 1984, S.81; Andreas Pfitzmann u. a. (Fn. 21). S. 118ff: Joachim Scherer (Fn. 31), S. 38 m. W. N.

S2 Auf elwa 350 Millionen Fernmelderechnungen cntfielen Im Jalir 1987 erwa 500000 und im Jalhr 1988 «twa 100000 Finwendungen, 2150 wensger als 2 bew. 0,5 Pronullc - 5. Ulyich Lange (Fn. 29), S. 11; Splegel 38/1989, vom 18.9. 1989, S. 259 .

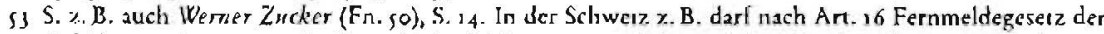
Gebührennachweis nus die Vomahl der Zielnummer enthalien, und in Frankreich müssen dic lecxicn ver Ziffern getilgr werden - s. z. B. FR vom 19.8.1989 sowic Hans-Peter Gebharde, Rechisgrundlagen des Datenschurzes sowne Datenschuiz im Fernmeldewesen der Iänder Schiveiz. Frankrisch, Niederlande, Großberiannicn. Schweden. USA und Japan, in: Jahrbuch der Deuschen Bundesposi 1987. 5.257. Das gleiche gilt, wenn in Nebenstellenanlagen Vorgeserzte die dienstliche Notruendigkert von Ferngesprächen nachprüfen wollen - ebenso z. B. Jorben Nungessur, Hessisches Daenschuticgesetz, Kommentar, Manz 1988, $\$ 34$ Rdnr. 47 : Landesbcauftragter für den Datenschutz der Freien Hansestadt Bremen, 11. Taugkertsberichn (Fn. $\left.4^{S}\right), S .46 ;$ s. hierzu auch BAG, BB 19\$7, S. 1037. Der Bundesbeauftragte für den Datenschuix regı un seinem 9. Täugkeıssberıche (1987), 5.93 an, Verfahren der Gebührenzählung und abrechnung beim 'Teilnehmer enzuführen.

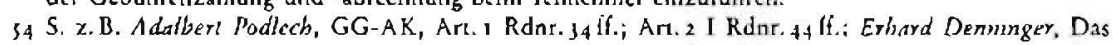

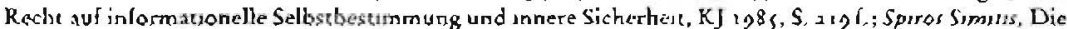
informationclle Selbstbestımmung, NJW 1984, S. 400 ; Cbnstoph Gusy (Fn. 16), S.96; A. A. Hans D. Jarass/Bodo Pieroth, Grundgesetx-Kommentar 1989, Art. 1, Rdnr.7.

ss E.benso LAG Hamburg, zustimmend zitier vom Landesbeauftragen für den Datenschutz der Freien Hansestadi Bremun (Fn. 48), 8 L, Jocben Nungesser (Fn. 53), \$34 Rdnr. 47: Wolfgang Schatzscimerder, Regiserierung des außeren Ablauls von 'reléfongesprachen - Eingriff oder immanente Schranke des Fernmeldegchumnisses?, ZRP 1981, S.1joff., Herbert Kubicek (Fn. 48 ), 5. 24 (; Chrutopls Gusy 
schender Meinung nur, soweic über den Kommunikationsvorgang oder -inhalt "Informationen 4 erhoben, gespeichert oder sonstwie verarbeirer werden. ${ }^{6}$ Die damic verbundenen Rechisprobleme werden bereits erkannt und - wentr auch noch viel zu schwach - diskutiert. Während sich das Recht auf informationelle Selbstbestimmung danach auf personenbezogene Daten als Kommunikationsinbalt bezieht und die Verfügungsbefugnis über dic Informationen gewährleister, die das soziale Bild des Einzclnen prägen", schüizt es nicht die Kommunikation als aktuellen Proze $\beta$ der Identitäisbildung und Selbstdarstellung.

\section{Das Recht auf kommunikative Selbstbestimmung}

Die Technik der Telckommunikation gefährdet die Pcrsónlichkeıtsrechte der Bürger jedoch nicht nur durch die mir ihr verbundene Datenverarbeitung. Sie greift in die Kommunikation von Menschen ein und fornt und bescimmt diese. Auch wenn es sehr schwer ist, die schleichenden Auswirkungen so geformter Telckommunikation in rechtiche Karegorien zu \{assen, darl diese Schwierigkeit nicht davon ablialten, nach Bewertungskriterien und Schutzmodellen zu suchen, um den erforderlichen Schurz der Persönlichkeit sicherzustellen. Diese Bemühungen sollten zunächst vor allem den Folgen gelten, die unmittelbar durch dic konkrete Arı der Kommunikationsvermitrlung verursacht werden und sich auf die Fntfaitungsfrciheit und den Persönlichkeitsschurz auswirken. Vor diesen Folgen vermag das informarionelle Selbstbestimmungsrecht nur unzureichend zu schützen.

Solche spezifischen Risiken der ISDN-Technik sollen an scehs bisher kaum problcmatisierten Leistungsmerkmalen aufgezeigr werden: dem automatischen Rückruf im Freifall, dem Heranholen, der Konferenz, dem Lauchören, dem Freisprechen und dem Direktansprechen. Ob die Risiken cassächlich in der beschriebencn Form beszehen, hängt schr stark von den Details der Ausgestaltung ab, die zwischen Anlagen verschicdener Anbicter differieren und zwischen Versionen eincs Systems Veränderungen unterliegen können. ss

(1) Beim automatuschen Rückeruf im Freifall hinterläße der Anrufer, der den angewähleen Teilnehmer nicht erreicht, obwohl dieser frei isı - cewa, weil er abwesend iss - durch Tastendruck oder Nachwahl einer Kennzilfer eincn Rückrufwunsch, der im System registrierc wird." Nachden der gerufene Teilnehmer wieder ein Telefon-

(Fn. 16), S. 96; Ulrcis K. Presß, Die Veranderung unserer Schnuerzgrenzen, Merkur 1996. S. 142; a. A. zur Notwendigkelt der Zustimmung des Angerufenen fur Nebenstcllenanlagen Hans Glius/Georg

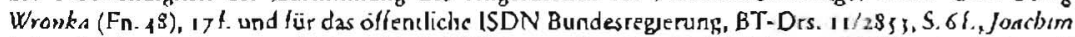
Schmidt (Fn. 48), S. 339; nach BayObLG, DVBl i974. S. $59^{8}$ und OVG Bremen, NJW 1980. S. 667 soll dic Wirksamker emes Verzichts suf das Fernmeldegehermnis niche davon abhängen, daß der Angerafene cbenfalls zustumnt; knusch herzu Wolfgang Schatzscbneuder (Fn. 33), S. 268 f.

s6 S. BVerfGE 65. 1 (43); nach Walter Schmule Glaceser (Fn. 43), S.85 m. w. N. Ist es sogar auf .Datco a in „Dateien " beschrankt; s. dagegen x. B. Cerhard Groß, Das Reche auf informationelle Selbstbesummung mie Blick auf dic Volkscahlung 1987, das neuc Bundesstatuscikgeset< und die Ameshilfe, AöR 1988 , S. 166, der fur den Grundrechessehuiz kencen Unterschied mathe, ob cine information manueli oder autornausch verarbertec wind.

57 Dies konnen auch Infomnationen über Kommunikatıonsprozesse sein.

s8 Vgl, zum folgenden ausführlich Volker Hammer/Ulracls Pordesch/Alexander Roßnagel(Fn. 22), S. 4 Iff. sowic Peler Kahl, ISDN. Das künftuge Femmeldenetz der Deutschen Bundespost, 2. Aufl. Heidelberg 1986; S. 235 ff.; Fernmeldetechnisches Zentralame, Teilnchmerbezogene Dienstmerkmale fur die Vermetelungsstelle, Feinspezifikatuon aus der Siche des Bcnutzers, Realisterung 1988, FTZ-Richilinic ITR 241 und Realisierung 1990, FTZ.Richelinic ITR 241, Ausgube $12 / 88 ;$ Hans-Peter Höller $(\mathrm{Fn}, 48)_{\text {; Peter }}$ Bocker (Fn. 17), S. 2 ff, und die Produkischrilten der Anbieser von ISDN-Anlagen.

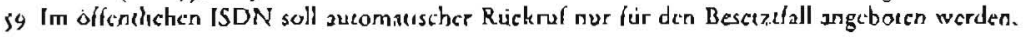


gcspräch geführt hat, wird nach dem Auflegen automatisch die Verbindung aufgebaut. Dabes ertont der Rufton zunächst beim Rufer und erst, wenn dieser den Hörer aufnimmc, wird dic Verbindung aufgebaut. Der Vorceil dieses Leistungsmerkmals liegt im Komfort für denjenigen Teihehmer, der den Gerufenen nicht crreichen kann und nicht mehrmals versuchen muß, diesen anzurufen.

Durch das Leistungsmerkmal kann jedoch zum einen die autonome Arbeitsgestaltung gefährdet werden. So können sich beispielsweise an *kommunikationsincensiven $\alpha$ Arbeitsplätzen mehrere Rückrufaufträge anhäufen. Wird eine längere Rückrufliste nach dem ersten Anruf wabgearbeitet $\propto$, so kann der Teilnehmer zu melureren Telefongesprächen in Folge gezwungen worden. Neben dicsem Nachteil besteht zum anderen das Risiko, daß von jedem rückrufberechrigren Anschluß aus unbemerkr Anwesenheitskontrollen an anderen Anschlüssen durchgeführt werden. Das Klingelzeichen signalisiert dem Rufer, daß der Gerufene scin erstes Gespräch seir Eintragung des Rückrufaufrages beendec hat - an vielen kommunikationsincensiven Arbeirsplätzen ein zuverlässiger Indikator für die (Wieder-)Aufnahme der Arbeit. Diese Koncrolle könnte etrua dort besonders wirksam werden, wo der Teilnehmer jeden Morgen den Hörer aufnehmen muß, um zu hören, welche Nachrichcen in scinem Sprachpostfach ,liegen. Der Angerufene scinerseits kann nicht erkennen, daß er kontrolliert wird. Nimnı nämlich der Rufer den Rückrul, der ihm in seinem Display oder durch einen besonderen Rufton signalisiert wird, nicht an, so kann der gerufene Teilnehmer nicht erkennen, daß ein Rückruf eingeleiter wurde.

(2) Die Leistungsmerkmale der besonderen Verbindungsvollendung, crmäglichen, daß Anrule, die sich an einen bestimmten Anschluß richten, einen anderen Anschluß erreichen. ${ }^{60} \mathrm{Mit} \mathrm{dem}$ Leistungsmerkmal Heranholen wird dem berechrigten Teilnehmer die Möglichkeit gegeben, einen Anruf, der an einen anderen Anschluß in einer festzulegenden Gruppe gerichret ist, durch Berätigen einer Taste an scinem Apparac cntgcgenzunchmen. Es bictet sowohl dem Rufer wie auch dem Gerufenen den Vortell, daß der Gerufene auch an einem anderen Arbeitsplatz Gespräche annehmen oder in seiner Abwesenheit ein Kollege das Gespräch heranholen kann.

Wie aber ist der Fall zu beurteilen, daß der Rufer ausschließlich den Geruíenen sprechen und vor anderen diesen Kontakt geheim halten möehte? Er kann nichs mehr darüber bestimmen, mit wem er Kontakt aufnimms. Wird ihm an einem Apparat mir Display angezeigt, daß er umgeleiter wurde, kann er zwar den Ruf abbrcchen. Tut er dies nicht, erhäht er sich die Chance, mit dem Gerufenen an cinem anderen Anschluß zu sprechen, geht aber zugleich das Risiko ein, daß ein Dritcer das Gespräch annimmt. Verfügt der Apparat, an den er umgeleitet wurde, cbenfalls über ein Display, wird dort, noch bevor er aufgelegt hat, seine Rufnummer - auch für weirere Personen sichtbar - mit einem Umleitungszeichen angezeigr. Besizz der Rufer gar nur ein saltmodisches. Analogtelefon, wird ihm die Umlenkung überhaupt nicht signalisiert.

Die Leisrungsmerkmale der besonderen Verbindungsvollendung، sind noch problematischer bei der Übertragung von Bildern, Texten odcr Daten, weil sich niemand am andcren Ende meldet. Ob dic Nachricht bei dem ankommt, der angewählt wurde, oder bei einem anderen Teilnehmer, kann der Rufer niclur crkennen. Ilum wird - wenn er über cin Display verfügr - zwar signalisier, daß die Verbindung umgelenkt wurde. Daraus kann er aber nicht ersehen, ob etrva der von ihm Gerufene die Verbindung zu einem anderen Anschluß nachgezogen hat oder ob ein Dricser die Verbindung - cventuell sogar gegen den Willen des Gerufenen - zu sich herangcholt hat.

Go Von diesen werden in offerilichen ISDN nur Anrufumlestung und Annifwetterschalrung angeboten. 
(3) Dic 1SDN-Tcchnik bietet die Möglichkeic, zu cinem Gespräch einen oder mehrere weitere Teilnehmer hinzuzuschalten. Dies kann allerdings nur derjenige, der das Gespräch eröffnct hat, indcm or wcitere Gesprächspartner hinzuwählt. Vorteil: Alle Formen der Konferenzschaliungen losen jedoch die tclefontypische Zweier-Beziehung auf. Daher ist es exforderlich, den an einer Konferenz Beteiligten Klarheit über die Kommunikationssituation zu verschaffen. Der Konfercnzleitcr hat zumindest dic Kennonis darüber, wen er zur Konferenz zusammengeschaltet hat. Für die anderen Teilnehmer der Konferenz ergibc sich jedoch das Problem, daß andere für sie unbemerkt hinzu- oder wcggeschaltet werden könnten.

(4) Verfügt ein Teilnehmer über cin entsprechendes Endgerär, kann er cinen Lautsprecher zuschalten und so das Gespräch hören, ohne den Hörer an scin Ohr halten zu müssen. Diesem Vorteil für den einen Teilnehmer stehen aber Nachteile für den anderen Teilnchmer gcgenüber. Laucbören machr das im Telefongespräch gcsprochene Wort anderen Menschen im jeweiligen Raum zugänglich. Die Vertraulichkeit des Gesprächs ist nicht mehr gewährleistet. Das Mithören anderer kann Telefonteilnehmer verunsichern, unter Umständen verändert die Kenntnis über das Mithören die Bereitschafr, bestimmte Inhalte zu diskutieren. Lauchören ist heure schon mir Zusatzciurichtungen nöglich. Der Kommunikationspartner kann über dic Akcivicrung der Lauthöreinrichtung in der Regel nur wissen, wenn er darüber im Gespräch informiert wird. Er ist also auf die Vertrauenswürdigkeit seines Kommunikationspartners angewiesen.

(s) Bercits heute verfügen Telefonapparate mit gehobener Ausstattung über eingebaute Mikrofone. Künfrig werden jedoch erheblich mchr Apparate mir der Möglichkeit zum Freisprechen ausgestatret sein. Wenn der Teilnehmer gleichzeitig noch Lauthören aktiviert hat, kann er das Gespräch bei aufliegendem Hörer führen und hat die Hände frei, un dabei gleichzeicig beispielsweise seinen Computer zu bedienen. Durch das Merkmal Fresprechen entsteht allerdings auch ein gewisses Abhörrisiko. So könnte jemand von einer Nebenstclle aus eine Verbindung zu einem anderen Raun aufbaven, Freisprechen akrivieren und dann den Raum verlassen. Sofern allerdings sichergestellt ist, daß der Anschluß-Inhaber den Status des Mikrofons leicht erkennen kann und autonom über scin Endgerät verfügt, ist das Risiko eines unbemerkten oder versehentlichen Abhörens sehr gering. Allerdings könnten weitere Personen, die sich gleichzeitig in diesem Raum aufhalten, betroffen seir, weil auch ihre Gespräche vom Mikrofon aufgenommen werden und am andercn Ende der Verbindung gehört werden. Bewußt oder unbewußt kann ihre Kommunikation mit diesem Leistungsmerkmal an den Telclonpartncr übermittelt werden.

(6) Ursprünglich war das Leistungsmerkmal Frcisprechen nur auf die jeweilige Nebenstelle begrenzt. In modernen ISDN-Nebenstellenanlagen sind jedoch Leistungsmerkmale vorgesehen, bei denen zugleich die Funktion einer Wechselsprechanlage in die Endgerätc und das Vermittungssystem integriert ist. Von dazu gceignetcn Telefongeräten aus können dann in anderen Endgeräcen mit geeigneter Ausstatung cin Mikrofon und ein Lautsprecher eingeschalrer werden. Diese Möglichkeir wird mit Drektansprechen bezeichnet. Der Rufer wählt dazu die Nummer seines Sekretärs und drückt dic Taste lür Direktansprechen. Das Telefon des Sekretärs klingelt einmal, dann sind das Mikrofon und der Lautsprecher der gerufenen Nebenstelle eingeschaitet.

Im Unrerschied zu Freisprechen wirke Dircktansprechen aul den Mikrofon- und Lautsprecherschater eincr anderen Nebenscelle. Je nach Qualität des Mikrofons können die Geräusche am Arbeitsplatz bis hin zum ganzen Raum erfaßc werden. Da dieses Mcrkmal durch die Zencrale oder andere Endgeräte (Chcfapparat) geschaltet 
wcrden kann, besteht eine Möglichkeit, die Umgebung der Nebenstelle abzuhören. Probleme können im Einzelfall insbesondere dann entscehen, wenn der Sckretär nicht autonom entscheiden kann, ob diese Funkuionen an seinem Endgerät aktiviert wird. Wenn zusätzlich nur eine unzureichende Signalisierung des Gerätestatus am Endgerät crfolgt, dann kann sein Arbeitsplat\% abgehörı werden.

Die bisherigen Konkretisierungen der Entfaltungsfreiheit und des Persönlichkeirsschuczes vermögen vor den angesprochenen Risiken nicht ausreichend zu schützen. $\mathrm{Da}$ in diesen Fällen keine Datenvcrarbeitung startfinder bzw. bcim automatischen Rückruf und der Rufumlenkung die interne Verarbeitung von Verbindungsdaten nicht das Problem darstellt, verhilft dem cinzelnen Teilnehmer die Befugnis, über die Speicherung und Verwendung seiner Daten selbst bestimmen zu dürfen, nicht zum Schutz seiner Kommunikation. Ebensowenig schürzt ihn das Reche am cigenen Wort, das ihm die alleinige Entscheidungsbelugnis darüber gibt, ob dieses mitgehört, festgehalten und verwendet werden darf. Denn von den Abhörmöglichkeiren des Lauthörens und Freisprectzens abgesehen, liegen dic Risiken in den genannten Beispielen vor allem in der mangelnden Transparenz und in der Fremdbestimmung der Kommunikationssituation.

Wie aber können diese Risiken für dic freie Entíalcung und den Scluutz der Persönlichkeir juristisch erfaßt werden? "Die bisherigen Konkretisierungen durch die Rechrsprechung umschreiben den Inhale des Persönlichkeitsrechts nicht abschließend. $\alpha^{61}$ Art. 2 Abs. I GG erlaube jedoch keine Lücken im rechelichen Schurz der menschlichen Freiheit unư Persönlichkcit. Vielmehr müssen die Lücken den neuen Risiken adäquat konkretisiert werden. ${ }^{62}$ Dabei können aus den bisherigen Ausprägungen Anhaltspunkte für eine kommunikationsbezogene Konkretisierung des Grundreclits aus Art. 2 Abs. I GG gewonnen werden.

Aus den Entscheidungen des $B V_{e r f} G$ zum allgemeinen Persönlichkeitsreche läßt sich nicht nur ableitcn, daß dieses cinen Anspruch auf Abschirmung einer privaten oder gar intinen Vertrauenssphäre gewährc. Vielmehr zielt der Schuzz der Persönlichkeit ebenso auf die Sicherung einer zencralen Voraussetzung für das Tärigwerden der Person in den Bezietsungen mit (niche vertrausen) Dritten und für das Tätigwerden in der Öffentlichkeit. In seinem sorialen und kommunikativen Kern bcinhaltet das allgemeine Persönlichkeitsrecht das Reche zur autonomen Selbstdarstellung. ${ }^{{ }_{3}}$ Es geht niche nur - wie bei der allgemeinen Handlungsfreiheit - um den Schutz einzelner Becätigungen, sondern um wesentliche Vorausseizungen dicser Berätigung. Man könnte davon sprechen, daß hier die ssoziale Identitär « geschuïzt wird, wälırend es bei der Abschirmung der Privat- und Incimsphäre um die nndividuelle Idencität" geht. ${ }^{\sigma_{4}}$

Eine den Risiken moderner Telekommunikation adäquate Konkretisierung ist in Anlehnung an die bisher gefundenen komnunikationsbezogenen Ausprägungen des Grundreches aus Art. 2 Abs. I GG zu suchen. Das Recht am gesprochenen Wort schützt ndie Befugnis..., sclbst zu bestimmen, ob seine Worte cinzig dem Ge-

61 BVerfGE 65, 1 (41): $54,14^{8}$ (153 f.); s. hierzu auch Waher Scbinu Glneser (Fn. 43), S. 88.

62 S. herzu \&. B. Waleer Schmite Glaeser (Fn. 43), S. 44.

63 S. hrerzu näher BVerfGE 65, I (41); Adalbert Podlech, GG-AK, Art. I Rdnr. 20, 39. Art. 1 Rdnr. 1;; ders., Das Reche auf Privathen, in: Joachins Perels (Hrsg), Grundrechte als Fundament der Demokrate, Frankfurt 1979, S. 51: Peter Saladin, Verantwonung als Stantsprinzip, Bern 1984, S. 203; Dietwald Roblf, Der grundrechtliche Schutz der Privatsphäre, Berlin 1980, S. 163 ff., Wilhelm Stemmüller u. a., Grundfragen des Datenschutzes, BT-Drs. 6/2836, Teil B, S. 47 ff., Teil C, S. 81 ff.

64 Hans D.Jarass, Das allgerneme Persönlichkestsreche im Grundgesety. NJW 1989, S. 899; s. hrerzu auch BVeriGE 54, ${ }_{4}$ \& (153): Walter Sebmut Glaeser (Fn, 43). S. 54, 39: Peter Schlechtriem, Inhalt und systematischer Standort des allgemeinen Persönlichkettsrechts, DRiZ 1975, S. 66; Hans Erıch Brandner. Das allgeneine Persönlichkeitsrecht in der Entwicklung durch die Rechtsprechung. JZ 1983.5 .690 . 
sprächspartner, einem bestimmen Kreis oder der Öffentlichkeit zugänglich sein sollen ess und nob und von wem seine auf einem Tonträger aufgenammenen Worte wieder abgespielt werden dürfen «. ${ }^{66}$ Es schüızt davor, daß dem Grundrechrsträger "Äußerungen in den Mund geicge werden, die er nicht getan has und die seinen von ihm selbst definiercen sozialen Geltungsanspruch beeinträchingen ". ${ }^{67}$ Das Recht am etgenen Bild schürzı "das Verfügungsrecht über Darstellungen der Person. Jedermann darf grundsätzlich selbst und allein bestimmen, ob und invieweit andere sein Lebensbild oder bestimmte Vorgänge aus seinem Leben öffentlich darscellen dürfenu. ${ }^{6 S}$ Das Recht auf informatuonelle Selbstbestummung gewährleiscer dic Befugnis, "grundsützlich selbst über die Presgabe und Verwendung seiner persönlichen Daten zu bestimmen ${ }^{69}$

Diese Konkrecisierungen können in vielen Falikonstellationen moderner Telekommunikation betroffen sein, gewährleiscen aber kcinen umíassenden Schurz gegen die Risiken des ISDN. Sie sind Ausschnitre brw. besonderc Ausprägungen des allgemeinen Pcrsönlichkeitsrechts ${ }^{70}$, umschreiben den Schutz der Persönlichkeit jedoch nicht abschließend. ${ }^{\prime \prime}$ Aufgabe des allgemeinen Persönlichkeitsrechts ist es viclmehr, die konstitutiven Bedingungen der Persönlichkeitsbildung jeweils adäquat zu schützen, "namentlich im Blick auf moderne Entwicklungen und die mis ihnen verbundenen neuen Gefährdungen für den Schurz der menschlichen Persönlichkeit.$^{72}$ Unter sich wandelnden Umständen kann der Persönlichkeirsschucz nur zielorientiert verwirklicht werden: Geschützt sind jeweils wdie Rechtspositionen, die für die Entfaltung der Persönlichkeit norwendig sind ".73

Materiell geht es in all den genannten kommunikationsbezogenen Ausprägungen um den »dem Schutz der Persönlichkeit zugrundelicgenden Gedanken der Sclbstbescimmung: Der Einzeine... soll grundsätzlich selbst cntscheiden können, wie cr sich Dritten oder der Ölfentliclukeit gcgenüber darstellen will, ob und inviewcit von Dritten über seine Persönlichkeit verfügt verden kanna.74 Die Selbsrbcstimmung seczt voraus, daß der von dem Einzelnen seibst definierte soziale Geltungsanspruch nicht beeinträchrigt wird und daß es "nur Sache der einzeinen Person sein (kann), über das zu bestimmen, was ihren sozialen Gelungsanspruch ausmachen soll k. "7

Dieses Selbstbesummungsrecht betrifft nicht nur die Entscheidung zu einer Kommunikation, in der sich der einzelne darzustellen vermag, und deren Inhal, sondern es muß auch die Kommunikarionsparener, den Kommunikationsort und die Kommunikarionsarr ${ }^{76}$ sowic das Kommunikacionsmedium umfassen. Ziel einer Konkrctisierung des Persönlichkeitsschurzes angesichts der Risiken der Telematik muß die Erhaltung von Konmunikationskomperenz sowie Entscheidungs- und Handlungskompetenz sein, die durch Selbstbestimmung über die Kommunikationssituation ermöglicht wird. Die Freiheit, über dic relevanten Umstände der Kommunikation

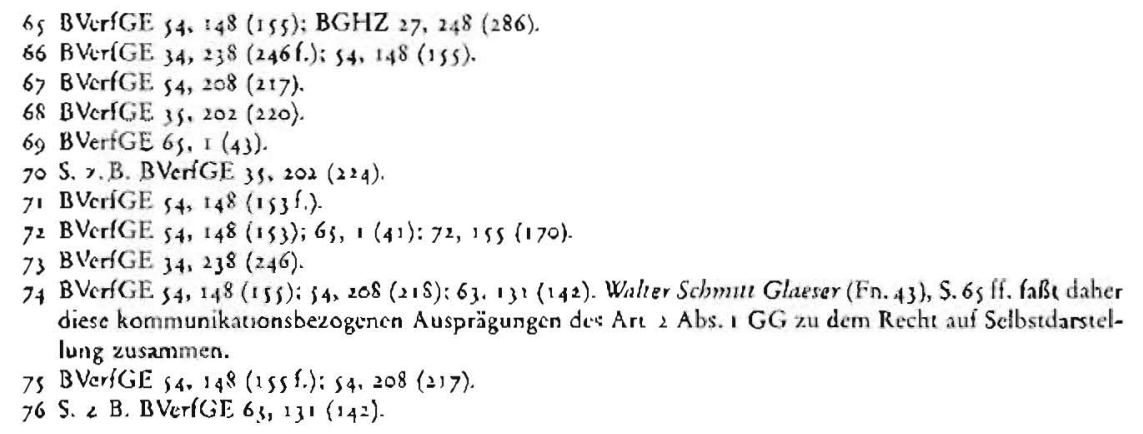


entscheiden zu können, muß in vergleichbarer Weise gewährleister sein wie in der unmittelbaren Kommunikation ohne technisches Medium. ${ }^{7}$

Die Kommunikatıonskompetenz als Schutzgut des Art. 2 Abs. I GG hat das Bundesverfassungsgericht in sciner Tonband-Entscheidung ancrkannt. Die crforderliche - Unbefangenheit der nicht-öffentlichen Kommunikation fehlt, wenn wein jeder mir dem Bewußtsein leben (muß), daß jedes sciner Worte, cine vielleichr unbedachte oder unbehcrrschte Äußerung, eine bloß vorläufige Stellungnahme im Rahmen eines sich entfaltenden Gesprächs oder eine nur aus einer besondexen Situation heraus verständlichc Formulierung bei anderer Gelegenbeit und in anderem Zusammenhang hervorgeholt werden könnte, um mit ihrem inhalt, Ausäruck oder Klang gegen ihn zu zeugen. Private Gespräche müssen geführt werden können, ohne den Argwohn und die Befürchiung, daß dercn heimliche Aufnahme ohne die Einwilligung des Sprechenden oder gar gegen dessen erklärten Willen verwerter wirde. ${ }^{\text {t }}$ Aus diеsеп Gründen sind sie nichr nur gegen Verdinglichung, sondern gegen jede ungenehmigtc Kennenisnahme zu schürzen. Daher ist zum Beispiel jede Überwachung des Telefons ein Eingriff in die geschürzte Kommunikation. 7

Der Schutz unbefangener Kommunikation ist nicht auf den abgeschlossenen $\mathrm{Be}_{-}$ reich einer ,Privatsphärec beschränkt, sondern crfaßt als Grundvoraussetzung persönlicher Idencitäısbildung auch die selbsıbestimmıc Kommunikacion außerhalb diescr Sphäre. ${ }^{30}$ Kommunikationskomperenz ist auch im Rahmen eines dienstlichen Gesprächs oder eincr dienstlichen Nachrichtenübermictlung zu gewährleisten. Denn sic sind nur für einen oder eine begrenzte Anzahl von Emplängern bestimmt und in diesem Sinn cbenfalls nicht-öffentliche Kommunikation. Die Möglichkeit, unbefangen zu kommunizieren, is zwar durch den dienstlichen Zweck beschränkt und überlagert. Dennoch ist es die individuelle Angclegenheit des jeweiligen Gesprächspartners, in welchem Stil cr kommuniziert, welche, Gesprächstaksik، er verfolgi, wie offene oder verschlossen er sich gibt - wie er sich und seinc Aulgabe seinem Gesprächsparıner präsenciert. Von *fernmünd\}ichen Durchsagen, Bestellungen oder Borsennachrichcen* abgeschen, für dic nder objekrive Gehalt des Gesagzen so sehr im Vordergrund (stehr), daß die Persönlichkeir des Sprechenden vollends dahinter zurücktritt $\alpha^{\alpha 1}$, muß daher die Unbefangenheit der Kommunikation grundsärzlich auch für dienstliche Gespräche geschüız sein. ${ }^{82}$ Daher beeinträchrigen auch heimliche Tonbandaufnahmen am Arbeitsplatz das Persönlichkcitsrecht, sofern der Betroffene auf die Vertraulichkeit der Äußerungen vertiauen durfte. ${ }^{3}$

In der Volkszählungs-Entscheidung wird als weitere Vorausseczung der Persönlich-

77 Ahnlich auch Christoph Gusy (En. 36), S. 90.

73 BVerfGE 34, $238(24,16)$.

79 S. BVerfGE 67, 157 (169); BAGE $40,37\left(4^{2}\right)$ - s. hicrau auch Gisulher Rispke, Der verfissungsrecheliche Schuix der Privatheiı, Baden-Baden 1976, S. 84

So S. hierzu für den betricblichen Bereich ausdrücklich BAGE 40,37 (41): s. allgemcin 7. B. Ernse Bended, Privassphare und, Personlishketsprofile, in: Festschrife für W. Geuger, Tübungen 1974, S. 34: Adalbere

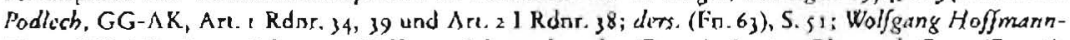
Riem, GG-AK. Are.s Rdnr. 15 ; Wolfgung Schataschnedder (Fn. 33), 5. 269; Christoph Gusy (Fn. 36). S.8gl: Hans Erscls Brandner (Fn.64). S.693 - lür ene ungenehmıgte Tonbandaufnahme in der öfentlichen Sitzung eines Gemeinderaes s. OLG Koln, NJW 1979, S. 661.

8. BVerfGE 34, 238 (247).

8: S. z. B. bereits Gunter Wiese, Der Persönliclikeisschul\% des Arbeienchmers gecenüber dem Arbcitgeber, ZIA 1971, S. $288 \mathrm{~m}$.w. N.. Hans Erich Brandncr (Fa.64), S.690; Hans Gliss/Ceorg Wronkia (Fn. 48). S. 20 bestummen dagegen den Schutzberesch des Rechts am gesprochencn Won zu eng.

si S. 2. B. BAGE 41, 37 (401.); BGH, NJW 1982, S. 1398 . Die Entschendungen des BGH NJW 1964. S. I65 und JR 19\$2, S.373, nach denen das Mithören eines Telefongesprächs mit geschältichem Inhalt auch ohne Wissen des anderen Teilnehmers im Geschäfts- und Wirtschaftsleben grundsáticl keine Verlezung des allgemeunen Persönlichkersrechis darstelle, widersprechen dem verfassungstechthichen Schutz der Kommunikation - s. z. B. BVerfGE 34, 238 (247); 67, 157 (169); BCHZ 73, 120ff.. OLG Koln, NJW 1979, S. 661 . 
keit die Freibeut zu selbstbestmmmler Entscheldung anerkannt. Individuelle Selbst-

bestimmung setzı... - auch unter den Bedingungen moderner Informacionsverarbeirungstechnologien - voraus, daß dem Einzelnen Entscheidungsfrciheir über vorzunehmende oder zu unterlassende Handlungen einschließlich der Möglichkeit gegeben ist, sich auch entsprechend dieser Entscheidung tatsächlich zu verhalten. Wer (dagegen) nicht mit hinreichender Sichcrheit überschauen kann, welche ihn betreffenden Informationen in bestimmicn Bereichen seiner sozialen Umwelt bekannt sind, und wer das Wissen möglicher Kommunikationspartner nicht einigermaßen abzuschätzen vcrmag, kann in sciner Freileit wesentlich gehemme werden, aus eigener Selbstbestinmmung zu planen oder zu entscheiden. ${ }^{\$_{4}}$ Un zu verhindern, daß die "Bürger nicht mehr wissen können, wer was wann bei welcher Gelegenheit über sie weißus', wird der Betroffene mit der Verfügungsbefugnis über sscine, Daten ausgestattet, muß die Datenverarbeitung bei ihm ihren Ausgang nehmen, ist ihm gegenüber legirimationsbedürfíg und muß ihm gegenüber transparent sein. ${ }^{k 6}$

Autonome Selbstdarstellung, Freiheit zur eigenen Entscheidung und zu ihrer Umsetzung, seczt Selbstbestimmung in der Kommunikation mit anderen voraus. Kommunikarive Kompetenz entscheider auch über die Entscheidungs- und Handlungskompetenz. Über die Möglichkciten zu kommunizieren darf daher nur unter Kennunis des Betroffenen und unter seiner Mirwirkung entschieden werden. ${ }^{87}$ Dieses Reche auf kommunikauve Selbstbestımmung schließr als norwendige Voraussetzung autonomer Selbstdarstellung nicht nur das Rccht cin, den Gesprächsinhalt, sondern auch dic Gesprächspartner und die Gesprächssituation frei zu wählen. Jede Kommunikation - auch zur Erfüllung dienstlicher oder arbeitsvertraglicher Aufgaben - dient grundsätzlich zumindest auch der individuellen Selbstdarstellung. Somic beinhalect dic durch Arr. 2 Ábs. $\mathrm{GG}^{8 \mathrm{~S}}$ gewährleistece kommunikative Selbstbescimmung das Recht cines jeden, selbst darüber zu bestimmen, mit wen er wann wo über welchen Inhalt und mitcels welchen Mediums kommunixieren will. ${ }^{89}$

\section{Kruerien zur Technikbewertung}

Um seine Wirkungen entíalcen zu können, bedarí das Grundrecht auf kommunikative Selbstbestimmung einer unterstützenden Ausgestaltung der Kommunikationstcchnik. Setzı diese dagegen »der Rechtsausübung so hohe Hindernisse entgegen, daß die Gefahr ciner Encwertung der materiellen Grundrechtsposition entsteht, dann ist (sie) mit dem Grundrecht, dessen Schutz (sie) bewrirken soll, unvarein-

\footnotetext{
$8_{4}$ BVerfGE 6 $\left.6,1(4\}\right)$.

S; BVeriGE 63, 1 (43)

86 S. hicrzu BVerfGE 65, 1 (63 (f.); Spiros Simies (Fn. 34), S. 400 ff., Erhard Denmuger (Fn. 54). S. 219 ff.. Adalben Podledb, Unter welchicn Bedingungen sind neue Informationssysicme gesellschaf(lich akzeptabel, in: Withelm Stenmüller (Hrsg.), Verdatet und vcrnetzi, Frankfun 1988, S. 122.

87 S. 2. B. auch Spuros Simums (Fn. 28), S. 46.

88 In Verbindung mil dem Schues der Menschenwürde und allen kommunikstıonsbezogenen Grundrechren. Diese betreffen jeweils Aspektr der kommunikauven Selbstbestummung und gehen insorvere dem Grundrecht aus Art. 2 Abs. I GG vor. Sie kónnen aber das Grundrecht aur kommunikatsve Selbstbestımmung nucht lückenlos ersetzen. S. etwa zur begrenzien Goltung des An.s Abs. I GG lür dic Telekommunikaton Joachm Scherer (Fn. j1). S. jo m.w. N.

89 S. hierzu auch Adalbert Podlech. GGi-A K. Are. 1. Abs. I Rdnr. 39, Art. 2 J Rdnr. 48 ; Chriszoph Gusy (Fn. j6). S. goll. Wolfgang Hoffmann-Riem, GG-AK, Art.s I Rdur. ss; ders., Massenmedien, in: Emst Benda/Werner Maiholer/Hans-Joclien Vogel (Hrsg.), Handbuch des Verfassungsreches, Berlin U. 7. 1983, S. 389 ff. Gisclher Rupke (Fn. 79). S. 81 ff.; Hans Enich Brandiner (Fn. 64), S. 690; Wilhelm

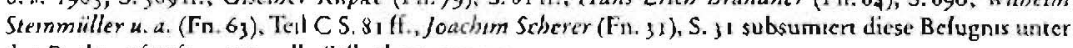
das Recht auf informationcllk Sclbabestmmung.
} 
barw.90 Nur wenn diese grundrechtskonforme Technikgestalcung gelingı, ist ein effektiver Schutz der Pcrsönlichkeit möglich. Das Recht auf kommunikative Selbstbestimmung kann allerdings nicht unmittelbar als Bewertungs- und Gescaltungsgrundlage an eine ISDN-Anlage herangecragen werden. Daher sind in einem ersten Konkretisierungsschritz aus dem Grundrecht Kriterien abzuleiten, denen eine ISDN-Anlage gerecht werden muß. Sie werden gewonnen, indem nach Regeln gesucht wird, nach denen die Anforderungen des grundrechelichen Schurzbereichs gegenüber den spezifischen Eigenschaften, Risiken und Anwendungsbedingungen der ISDN-Technik erfüllt werden können.

\subsection{Transparenz}

Um das Grundrecht ausüben und sich gegen rechtswidrige Eingriffe wehren zu können, muß die Kommunikationssituation für alle Bctroffenen transparent scin. Soll eine selbstbestimmte und unbefangenc Kommunikation gewährleistet sein, müssen alle Partner rechtzeinig darüber informierr scin, wer in welcher Form an dem Kommunikationsvorgang teilnimmt.

\subsection{Entscheidungsfreibeut}

Wie bereits gezeigr, serzc kommunikative Selbstbestimmung die Freiheit dcs Einzelnen voraus, sich zu entscheiden, welche Kommunikationshandlung cr wic wo wann und mit Hilfe welchen Mediums er vornchmen möchce. Niemandem darf daher gegen seinen Willen eine Kommunikationsbeziehung sufgenötigs werden. Alle Randbedingungen (Kommunikationspartner, -zeit und-umstände) müssen daher so rechtzeitig signalisiert werden, daß alie Beteiligten die Möglichkeit haben, auf die spezifische Situation zu reagicren.

\subsection{Erforderlichkent}

Jede aufgrund ciner Einwilligung oder eines Geserzes zulässige Bccinträchrigung ist auf das unbedingt erforderliche Mindestmaß zu beschränken, das zur Erfüllung der geserzlichen, dienstrechelichen oder arbeitsvertraglichen Aufgabe und zur Erreichung des konkreten Zwecks notwendig ist. Eine Grundrechtsbceinträchtigung, dic über dieses Mindestmaß hinausgeht, ist rechtswidrig. In der Direktruf-91 und in der Volkszählungs-Entscheidung ${ }^{22}$ hat das Bundesverfassungsgericht Grundrechrsbescluränkungen im Interesse des Allgemeinwohls akzepricrt, weil cine geeignete Technik, die diese vermeiden und dennoch den gewünschten Zweck erreichen könnte, nicht zur Verfügung stand. Dies heißt aber nicht, daß die Betroffenen alle Grundrechtseinschränkungen hinzunehımen hä̈ten, die sich durch die technischen Gegebenheiten der derzeit vcrfügbaren ISDN-Technik und deren Mängel ergeben. Eine solche Interpretation würde die Grundrechre einem Vorbehalt technischer Möglichkeiten ausseczen. ${ }^{95}$ Aus diesen Entscheidungen läßt sich im Gegenteil

90 BVerfGE 63, $131(143)$ - das Zira betrift in Origunal das Verfahrensrecht, ise aber sowolsi von seincen Worlaut und als aucls seinem Sinn her zul die Technikgestaltung übeniagbar.

9r BVeriGE ${ }_{4} 6,120(1 ; 21$,$) .$

92 BVeriGE 6s, 1 (ss).

93 So aber sind offenbar die Ausfubrungen von Hass Chiss/Georg Wromka (Fn. 48), 84. is zu verstehen. 
entnchmen, daß die Rechtfertigung für den Grundrechtseingriff entfällt, wenn dic Technik auch anders gestaltet werden könnte oder wenn gar grundrechrskonformere Techniken verfügbar sind. Die Beeinträchtigung ist dann nicht mehr erforderlich. Ein technisches Kommunikationssystem dart danach nur insoweit genuizt werden, als durch rechnische und organisatorische Verbesserungen die Grundrechtseinschränkungen nicht mehr weiter reduziert werden können. ${ }^{94}$

\subsection{Werkzengetgnung}

Selbschestimnt kann der Umgang mit der Kommunikationstechnik nur sein, wenn sie die autonome Arbeirsgestaltung möglichst wenig einengt. Dies setzt voraus, daß der Nurzer in der Lage sein muß, sie in ihren Funkcionen zu durchschauen und zu beherrschen. Er muß wissen können, unter welchen Bedingungen er kommuniziert, und $\mathrm{cr}$ muß in der Lage sein, diese Bedingungen zu bceinflussen. Insofern berühren sich die Kriterien der Werkzeugeignung, der Transparenz (4.1) und der Entscheidungsfreiheit (4.2). Dic Technik ist umso werkzeuggeeigneter, je weniger technische Sacharänge sie enthält. Sie muß ihm ausreichende Frciheitsgrade in der Organisation seiner Kommunikation und seiner Arbeitsabläufe bieten. Gegen diese Forderung spricht nicht, daß der Nutzer im Rahmen von Dienst- oder Arbeitsverhältnissen diese Spielräume of gar nich hat. Denn in diesen Fällen verlagert sich das Autonomieproblem nur auf cine andere Hierarchieebene. Denn dann muß die Technik es $\mathrm{dcm}$ Bescläftigten ermöglichen, Weisungen seines Vorgesetzcen zu befolgen. Soweit ihn daran technische Sachzwänge hindern, wird dadurch die Arbeitsautonomic des Vorgesetzten becinträchtigt. Über das $\mathrm{Ob}$ und das Wie einer Kommunikacion darf also - im Rahmen der Leistungsfähigkeit des Systems - nur der Nutzer, niche jedoch dic Technik bestimmen.

\subsection{Kontrolleignung}

Ëin effektiver Schurz des Rechts auf kommunikative Sclbstbestimmung setzt voraus, daß eine Beeinträclatigung dieses Rechts erkannt und bescitigt werden kann. Dic zuständigen Kontrollorgane müssen kontrollieren können, inwieweit das technische System dieses Grundrecht gewährieister, gefährdet oder verletzt. Eine wirksame Kontrollc dient eincm vorgezzogenen Rechtsschutz." Sie setzr jedoch voraus, daß alle sensitiven Aktivitäten innerhalb des Systems unveränderbar registriert werden und dicse Kontrolldaten den Kontrollorganen in übersichtlicher Weisc zur Verfügung gestcllt werden.

94 S. z. B. Wolfgung Schalyschiezder, Fernmeldemonopol und Veriassungsrechs, MDR 1988. S. s\}2: Hans Bronckmann, Telekommunikationsordnung und Fernmeldebenutxungsrecht, CR 1989, 5.97: Jocijen Nungesser (Fn. 53). \$34 Rdnr. 12. Das Krsterium der Erfarderlichkeit beziche sich jedach nich nur auf das, Wice, sondern auch aul das, Obe der Anivendung von Kommunikationstechnologien. Hier wie für jede grundrechisnskane Technikanwendung muß die Erforderlichkeı in ihrem Verbältnıs zum Allgemennwohl und zur Gefährdung der genannten Grundrechte geprüft werden - s. z. B. Adalberl Podiech, Individualdatenschutx - Systemdacenschurz, 1n: K. Brückner/G. Dalichau (Hrsg.), Beıträge zum Sozıalrecht, Festgabe f. H. Grüner, Percha 1982, S. 455 . In diesem Zusammenhang wären die in Fn. 21 genannten Systemalternativen zu diskutseren.

95 So z. B. BVerfGE 65, $1(66)$ und Erhard Denninger (Fn. 54), S. 1.28 für die Datenverarberung: für Telefonanlagen s. insb. Volkey Hanmey/Alexander Roßnagel. Datensicherung in ISDN-Telelonanlagen, DuD //1990. 
Aus diesen Kriterien lassen sich aber unmittelbar noch keine Aussagen herleiten, die cine rechuliche Bcwercung von ISDN-Systcmen ermöglichen. Daher isc in einer wciteren Konkrctisierungsscufc nach Zielen lechnuscher Gestaltung zu fragen, um ein ISDN-System kriteriengerccht auszulegen. Dic Ziele müssen so präzise sein, daß dic aus rcchtlicher Sicht erforderliche Ausgestaltung quasi plichtenheftgerecht beschrieben werden kann. In einer dritren Stufe sind dann konkrete technusche Auslegungsbedingungen zu formulieren, die erfüllt sein müssen, um die technischen Gescaltungsziele zu erreichen. Auf dieser Ebenc technischer Konkretion kann dann durch cinfachen Vergleich zwischen den Micrkmalen eines gegebenen ISDN-Systems und der geforderten Auslegung bewertes werden, wo dic Anlage rechtlichen Anforderungen entspricht und wo sie Defizite aufweist.

Die Kriterien ermöglichen durch diese Konkretisierungsschritte cine umfassende Bewertung aller grundrechtsrelevanten Aspekte von ISDN-Systemen. ${ }^{96}$ Wre technische Gestaltungsziele aus den rechtlichen Kritericn entwickelt und zu technischen Auslegungsbedingungen operationalisierı werden können, soll hier am Beispiel der Anruferidentifizicrung sowie der sechs weiteren bereits vorgestellten Leistungsmerkmale gezeige werden:

Das Problem der Auruferidentifuzierung wird erst dann richeg verstanden, wenn cs als ein Konflikt zwischen zwei Grundrcchten erkannt wird. Dieses Leistungsmerkmal greift zwar in das Recht auf informationclle Selbstbestimmung des Rufers ein, erfüllı allerdings gleichzeirig die aus dem kommunikativen Selbstbestimmungsrecht des Gerufenen folgenden Anforderungen auf Transparenz (4.1) und Entscheidungsfreiheit (4.2). Aufgabe der Kommunikationstechnik muß es sein, praktisclic Konkordanz herzusccllen, indem es jedem der Grundrechre zur oprimalen Verwirklichung verhilift.

Dieses Zicl könnre besser als durch die oben genannten Lösungen"7 durch ein *Handshake-Verfahrene crreicht werden: Der Gerufene fordert - solern er dies wünscht - durch einen Tastendruck die Identifikation an und der Rufer kann dannsofern er damit einverstanden ist - ebenfalls per Tastendruck die Übermitulung seiner Rufnummer freigeben..$^{8}$

Die technischen Gestaltungszicle des automatıschen Rückrufs ım Freifall müssen sich an den Kriterien der Transparenz (4.1), Entscheidungsfreiheit (4.2), Erforderlichkeit (4.3) und Werkzeugeignung (4.4) orientieren. Der Gerulenc muß den Rückrufwunsch erkennen und beeinflussen können. Er muß in der Lage scin, die Rückrufwünsche umzusortieren und unerwünschte Rückrufaufträge zu löschen. Außerdem muß er autonon über die Aktivierung des Merkmals watomatischer Rückrufu für seinen Anschluß entscheiden können und in der Lage sein, die Rückrufwünsche für scinen Anschluß «u begrenzen. Aber auch der Rufer sollte frei über die Spcicherung seines Rückrufwunsches enzscheiden und eingeleitete, aber nicht mehr gewünschtc Rückrufe aufheben können.

Um diese Ziele erreichen zu können, sollte ein ISDN-System folgende reclunische Eigenschaften aufwcisen: Das Leistungsmerkmal sauromatischer Rückruf im Beserzifall kanı unabhängig vom sautomatischen Rückruf im Freifall, inscallierr werden. Es ist möglich, Berechrigungen für die Rückrufeinleirung zu vergeben. Der

96 S. herzu näher Volker Hummer/Ulyich Pordesch/Alexander Roßnngel (Fn. 22), $41 \mathrm{ff}$.

$97 \mathrm{~S}$. obar ba Fn. 49 und so.

98 Die gleıche Forderung erhebt die französische Datenschurzkommission - s. Hans Precy Cebburdi (Fn. 53$) . S=67$. 
Rufer entscheidet per Tastendruck oder Kennzahlwahl über die Aufnahme seines Wunsches in die Liste für aucomarische Rückrufe und kann diesen aul die gleiche Weise wieder löschen. Nach einer bestimmten Zeit wcrden Rückrufwünsche automatisch gelösch. Der Gerufene kann an scinem Endgerät die Anzahl möglicher Rückrufe begrenzen. Er kann die Liste der Rückrufaufträge an seinem Display einsehen, umsorrieren und löschen. Dic Aktivierung des Rückrufs erfolgı durch den Gerulenen.

Die Gestaltung des Leistungsmerkmals Heranbolen hat sich an den Kriterien Transparenz (4.1), Entscheidungsfreiheit (4.2) und Erforderlichkeit (4.3) auszurichren. Ein Gespräch darf nicht gegen den Willen eincs Gcrufenen "wcgageholt werden könnelr. Personen mit (partiell) gegensäczlichen Interessen dürfen nicht in der Lage sein, in den Verbindungsaufbau des anderen einzugreifen. Zusätzlich sollte jeder Teilnehmer selbst darüber entschciden könnes, ob er das Merkmal für seinen Anschluß ́reigibu. Für alle Berciligten muß erkennbar sein, daß eine Rứumlenkung scatcfindet. Sowohl der Rufer als auch der Heranholende sollten frei über die Anzeige ihrer Rufnummer aul dem Display des Partners entscheiden können. Zumindest muß der Rufer die Möglichkeic haben, den Verbindungsaufbau abzubrechen, bevor seine Rufnummer aul dem Display des Heranholenden erscheint.

Um diese Ziele zu erreichen, isc folgende technische Realisierung anzustreben: Das Heranholen ist nur in Nebenstellenanlagen innerhalb bescimmitcr Gruppen möghich. Dicse werden im Rahmen der Bcrechnigungsvergabe definiert. Der NebenstellenInhaber kann das Merkmal autonom (de)akuvieren. Beim Verbindungsaufluau wird die Umlenkung signalisiett - entweúer am Display oder durch besonderen Aufmerksamkeitston - mir Abbruchmöglichkeir für den Rufer. Die Freigabe der Rufnummer effolgt auf beiden Seitcn im Handshake-Vcrfaluren.

Um die Transparenz (4.1) und Entscheidungsfrciheit (4.2) für alle Beteiligten an ciner Telefonkonferenz zu gewährlcisten und die Begrenzung ihrer kommunikativen Selbstbestimmung auf das Erforderliche (4-3) zu reduzieren, ist es erforderlich, $d_{a} ß$ allen die Konferenzsituation signalisiert wird. Zusäczlich muß das Hinzukommen bzw. Ausscheiden von Teilnchmern mit unterschiedlichen Tönen signalisiert werden, damir sich jeder - zumindest per Nachlrage - ein Bild über die Zusammenseczung der Konferenzgruppe machen und darauf reagieren kann. Das Leiscungsmerkmal isc daher so zu gestalten, daß immer dann, wenn ein Teilnehmer zur Konferenz hinzugeschaltet wird oder diese verlaßßt, dies allen Beteiligten akustisch signalisiert wird. Hierfür werden zwei verschiedene Signaltöne benurzıt. Dabei wäre zu wünschen, daß die Nummern der zugeschalreten/akriven Nebenstellen für alle Teilnehmer gemäß den Anforderungen an das Leistungsmerkmal identifizieren, abrufbar sind.

Die ISDN-Technik bieter im Gegensalz zu heutc die Chance, den entsprechenden Status der aktivierten Endgeräte allen Gespräclaspannern zu signalisieren. Damit könnte deren Vertrauen in die Kommunikationsbeziehung erlsöht werden. Sie sind nichr mehr von der Vertrauenswürdigkeit des anderen Teilnehmers abhängig. Ihre Entschcidungsfreiheit (4.2) kann aufrechterhalten, die Transparenz der Kommunikarionssituation (4.1) gefördert und die Werkzeugeignung (4.4) erhöht werden. Tcchnisch sind diese Kriterien erfülit, wenn Lautbören, Freisprechen oder Dirckiansprechen allen Beteiligten signalisicrt wird und nur von den Betroffencn ein-oder ausgeschaltet werden kann. Direktansprechen ist außerdem auf klcine soziale Gruppen zu begrenzen.

Diese Gestaltungsziele wären technisch dadurch zu gewährleisten, daß bei Lauthören bzw. Freisprechen das Mikrofon oder der Laursprecher nicht über das ISDNSystem, sondern nur am jeweiligen Endgerät ein- und ausgeschaltet werden kann. 
Sind sie eingeschaltet, wird der jeweilige Status am Endgerät durch eine deurliche oprische Anzeige angezeigt, die vom Raum aus für alle Anwesenden zu erkennen ist. Außerdem sollte in gewissen Zeitabständen ein Aufmerksamkeitston gesendet werden. Für Direktansprechen wäre außerdem techniscl zu gewährleisten, daß dic Berechtigung zur Aktivierung auf kleine Nebenstellen-Gruppen begrenzt werden kann. Die Freigabe einer Nebenstelie für das Direktanspreclen darf nur vom Endgerär des Gerufenen aus möglich sein. Sie kann jederzeit geseczt und widerrufen werden (Ansprechschuiz).

\section{Rechtlich bestimmte Technikgestaltung als Aufgabe}

Das Reche auf kommunikarive Selbsebescimmung kann durchgeserzt werden, indem es im Fall ciner Verletzung eingcklagt wird. Hierfür wärc in Einzelfall zu klären, ob das Grundrecht als Abwehrreche, Teilhaberecht oder Schutzanspruch fungierr. Denn diese Funktionsbescimmung lä̈ngt davon ab, wic in der jewciligen sozialen, durch die Technik und die okonomischen Randbedingungen geprägten Situation das Schuczziel des Grundrechts ratsächlich erreicht werden kann. ${ }^{99}$ Ebenfalls klärungsbedürfrig ist die Frage, uncer welehen Vorausscezungen dieses Grundrechr eingeschränkz werden darf, ob dies - etwa analog zum Recht auf informationclle Selbstbestimmung - nur durch Gesetz möglich ist, das diese Möglichkeit präzis und zweckspezifisch eröffner.

Wird eine Verletzung des Rechts auf kommunikative Selbstbestimmung allerdings erst gerichtlich geltend gemacht, beeinflußt die Macht rechnischer Systemzwänge und milliardenschwerer Investitionen die Rechesfindung. ${ }^{100}$ Für die Grundrechrsdurchsetzung viel sinnvoller ist daher der Weg der frïhzeitigen Technikgestaltung. ${ }^{\text {or }}$ Start sich mit dem idcologiekritischen Hinweis zufricdenzugeben. daß aus strukturelien Gründen das Recht im Scharten der Technik stehe ${ }^{102}$, ist nach Wegen zu suclien, wie Technik nach rechtlichen Kriterien gestaltet werden kann. Gerade die Kommunikationstechnik ist hicrfür ausreichend flexibel, sofern die Gescaltungsbemühungen frühzeitig cinsetzen. Wenn in das Techniksystem bereits investiert wurde oder es sogar schon entwickelt ist, läuft rechtrliche Technikgestaltung Gefahr, an den bereits formierten Interessen zu scheitern. ${ }^{103}$ Grundrechtsgemäße Technikgestaltung ist schwierig, aber nicht aussichtsslos. Dieses Vorhaben kann gelingen, wenn folgende Kritcrien erfüllt sind:

- Erstens muß das Wissen, welche Risiken ein Techniksystem für Grundrechre hervorrufen kann, so frühzeitig verfügbar sein, daß rechrliche Gestaltungsanforderungen noch entwickelt und durchgesetzt werden können.

- Zweitens sind zu jedem Entwicklungsansatz für cin Techniksystem technische und organisatorische Alternativen zu idenrifizieren.

99 S. x. B. Adalbert Podlecin, GG.AK, Art. 2 Abs. I Rdnr. 33.

io S. hierru ausführlich Alexander Roßnagel/Petir Wedde/Volker Hammer/Uirad Pordesch (Fn, I6).

10r Ebenso z. B. Hans Bnnckmann (Fn. 27), S. 44 f, Whibelm Sienmiller, Sozizle Beherrschbarket: offener Neuze, OVD-Online 10/198s, S. 149f.; Stefan Waly. Datenschutz be1 Telematikdicnsien, in: Joachum Schurer (Hrsg.), Telckommunikatıon und Wirtschaftsrectit, Köln 1988, S.217: Adalbcre Podlech (Fn. 63), S. 4 s Iff.; Joachism Scherer (Fn. 11), S. is I ff.; Hans-Joachum Kerkau (Fn, 11), S. zof.; Herbert Kubicek, Soziale Bcherrschbarkert icclinisch offener Netze, in: Rüdiger Valk (Hrsg.), Gl-18. Jahresenpung. Heidsiberg $1988,5,109$ ff.

102 S. 2. B. Ramer Wolf, Das Recht im Schanen der Technik, KJ 1986, S. 24 iff.

to3 S. hicru naher Alexander Roßnagel, Möglichkenten verfassungsvertaglicher Technikgestaltung. נח: ders. (Hrsg.), Frciheie im Griff, Informationsgesellschaft und Grundgesce, Suugart 1989, S. 177 if. 
- Drittens ist ein entsprechendes rechtliches Instrumentarium für die Bewerzung dicser Alternariven und das Hervorbringen von Gestalsungszielen zu entwickeln.

- Vierzens sind diese rechtlichen Kriterien bis hin zu ganz präzisen technischen Gestalungsaníorderungen zu konkretisieren.

- Und schließlich ist die Bereitschaft und die Macht erforderlich, diese Anforderungen gegen widersereicende Interessen auch durchzuscizen.

Hier wurde gezeigt, daß für cinige Risiken von ISDN die dritre und vierre Bedingung erfüllt werden kann. ${ }^{104}$ Die Chancen, die erste und zweitc Voraussetzung zu erreichen, sind bereits weitgehend vertan. Die Planungen für das öffentliche ISDN und entsprechende Normungsbemühungen sind schon sehr weic fortgeschritten. Allcrdings befinder sich die Verwirklichung erst noch am Anfang. Hier dürften daher noch einige Korrekcurmöglichkciten gegeben sein. ISDN-Nebenstellenanlagen sind zwar ebenfalls bercits entwickelt und schon vielfach installiert. Aber sie werden ständig weiterentwickel und bci ausrcichendem Druck oder besserer Einsicht möglicherweise in eine grundrechrskonforme Richrung geführt.

Die leczte Bedingung ist ebenfalls für Nebenstellenanlagen leichter zu gewährleisten. ${ }^{105}$ Im Bereich der Deutschen Bundespost sind die polizischen Einwirkungsmöglichkeiten seit ihrer Strukturreform erheblich schlcchter geworden. ${ }^{100}$ Dagegen können Becriebs- und Personalräte bei der Einführung von ISDN-Nebensielienanlagen im Rahmen des Betricbsverfassungs- oder Personalvercrecungsrechts eher durchsctzen, daß diese grunörechtskonform gesıaltet werden. Vielleicht aber wird der notwendige Druck zur vorbeugenden Technikgestalcung doch nur dann entaltet werden können, wenn er durch nachträgliche gerichtliclse Überprüfung von ISDN-Systemen verstärkt wird. ${ }^{107}$

104 S. hierzu näher Volker Hammer/Ulnch Pordesch/Alexander Rofßnagel (Fn. 22).

105 Ermuugende Beispiele hierfür könnten seın, daß Empfehlungen zur Technikgestalung, ivıe sı hıcr entwickelt wurder, aufgrund einer Beratung durch Hans-Peter Höller und Herberi Kubicek im Bundesiorschungs- und Bundesjustizministerium und aufgrund ciner Beraeung durch dic ,Projektgruppe verfassungsverträgliche Technikgestaltung (prover) in der Hochschulnegion Darmstade Berücksicheigung gefunden haben. - S. zum GestaltungsprozeB in zweiten Beispicl Vollecr Hammer/Ulnch PordeschiAlrxander Roßnagel, Rechesgemäße Gestalıung von ISIDN-Nebensicllenaniagen, in: M. Paul (Hrgg.), GI-19. Jahrestagung, Computergestützter Arbeitsplatz, Berlin u. a. rg\$, Band II, S. 307 fr.

106 S. hicru z. B. Alexander Roßnagel/Peter Wedde, Die Relarm der Deutschen Bundespose im Licht des Demokratieprinzips, DVBI 1988, S. 962 ff., Helmut Fangmann. Verfassungstechiliche Rabmenbedingungen der Telekommunikatıon, RDV 1988, 5. 53 ff., Krut Bach/Emst Hustadt, Die ,Deregulierung der Deutschen Bundespost, DuR 1989, S. 294 /f., Wolfgang Soljatxschneidey. Die Neustrukruncrung des Pose- und Fernmeldewesens. NJW 1989, S. $237 \mathrm{ff}$.

107 Wenvolle Hinwesse verdanke ich Johann Bizer, Volker Hanmer, Herber Kubicek, Ulnch Pondesch und Perer Wrdde. 